To appear in Statistics: A Journal of Theoretical and Applied Statistics

Vol. 00, No. 00, Month 20XX, 1-24

\title{
Strong laws for associated random variables
}

\author{
Tonguç Çağın and Paulo Eduardo Oliveira* \\ CMUC, Department of Mathematics, University of Coimbra, 3001-501 Coimbra, Portugal
}

(June 1, 2016; March 27, 2017)

\begin{abstract}
We study the almost sure convergence and rates of weighted sums of associated random variables under the classical assumption of existence of Laplace transforms. This assumption implies the existence of every moment, so we address the same problem assuming a suitable decrease rate on tail joint probabilities which only implies the existence of finitely many moments, proving the analogous characterizations of convergence and rates. Still relaxing further the assumptions on moment existence, we also prove a Marcinkiewicz-Zygmund for associated variables without means, complementing existing results for this dependence structure.
\end{abstract}

Keywords: almost sure convergence; association; convergence rates; exponential inequalities; Marcinkiewicz-Zygmund law

AMS Subject Classification: 60F 15

\section{Introduction}

Many useful linear statistics are written as weighted sums of random variables, raising thus the interest in the characterization of the asymptotics of such sums after a conveniently chosen normalization. Examples of such linear statistics include weighted empirical distribution functions, least-squares estimators, nonparametric regression estimators, here with random coefficients, or common predictors in linear time series models. Since Baum and Katz [1] proved that for $X_{n}, n \geq 1$, i.i.d. and centred, $n^{-1 / p}\left(X_{1}+\cdots+X_{n}\right) \rightarrow 0$ almost surely for $p \in(1,2]$ if and only if $\mathrm{E}\left|X_{1}\right|^{p}<\infty$, many authors studied this problem. Chow [2] and Cuzick [3] obtained conditions for the convergence for weighted sums $n^{-1 / p}\left(a_{n, 1} X_{1}+\cdots+a_{n, n} X_{n}\right)$ with independent random variables, later extended by Cheng [4], Bai and Cheng [5], or Sung [6], linking the moment assumption with the properties of the weighting sequence. Controlling the asymptotics of weighted sums often means that we may need the existence of moments of order larger than 2 , depending on the decrease rate of the weights.

The convergence of weighted sums of random variables has, naturally, also been considered dropping the independence assumption. In recent years we have seen several contributions considering negatively associated variables, or some other form of negative dependence. Here, we will be interested in positively associated variables. This later dependence notion raises distinct difficulties on the control of the variance of sums, often a crucial step for the proof of asymptotic results. Indeed, for positively associated

*Corresponding author. Email: paulo@mat.uc.pt

This work was partially supported by the Centre for Mathematics of the University of Coimbra UID/MAT/00324/2013, funded by the Portuguese Government through FCT/MEC and co-funded by the European Regional Development Fund through the Partnership Agreement PT2020. 
variables, the variance of sums is larger with respect to what is found for independent variables, while negative dependence has the opposite effect. A first result concerning the convergence of $n^{-1 / p}\left(X_{1}+\cdots+X_{n}\right)$ for positively associated and strictly stationary $X_{n}$ 's was proved by Louhichi [7], requiring the existence of low order, less than 2, moments. This has been, more recently, extended for weighted sums in Oliveira [8] and Çağın and Oliveira [9], using an approach similar to Louhichi's [7]. Here we consider results similar to those in $[8,9]$ but now based on the method used in Ioannides and Roussas [10] and Oliveira [11] for the proof of exponential inequalities. This approach means that we will find conditions for the almost sure convergence and also for its rate. These conditions depend on the covariances, thus require the existence of moments of order at least 2 , and link the exponent $p$ with the behaviour of the weighting coefficients, as for the characterizations for independent variables mentioned above. A classical assumption, even for independent variables, providing the control of moments requires of existence of Laplace transforms. We try to avoid this kind of assumption, as it implies the existence of finite moments of every order, and replace the existence of Laplace transforms by a polynomial decrease rate on joint tail probabilities, which only implies the existence of finitely many moments.

Finally, for random variables without means or stationarity assumptions, we prove a Marcinkiewicz-Zygmund strong law that complements earlier results by Chandra and Ghosal [12], Louhichi [7], Oliveira [8] and Çağın and Oliveira [9]. Again, assuming a suitable decrease rate on the joint tail probabilities, we prove a simple version of this strong law.

The paper is organized as follows. Section 2 describes the framework and some useful preliminary results, Section 3 describes the conditions for the almost sure convergence of weighted sums and its rates for bounded variables. This is a tool for the results proved in Sections 4 and 5, that are concerned with the cases of variables with infinitely or only finitely many moments, extending the characterizations for the almost sure convergence and its rates. Finally, Section 6 proves some general convergence characterizations for sums of associated random variables and a Marcinkiewicz-Zygmund law for associated variables without means.

\section{Definitions and preliminary results}

Assume that $X_{n}, n \geq 1$, are centred and (positively) associated random variables, that is, are such that $\operatorname{Cov}\left(f\left(X_{1}, \ldots, X_{n}\right), g\left(X_{1}, \ldots, X_{n}\right)\right) \geq 0$, for every $n \geq 1$ and coordinatewise increasing functions $f, g: \mathbb{R}^{n} \longrightarrow \mathbb{R}$ for which the covariance exists, and denote $S_{n}=X_{1}+\cdots+X_{n}$. Let $a_{n, i}, i=1, \ldots, n, n \geq 1$, be nonnegative real numbers and, following $[3,5,6]$ and many other authors, define, for each $\alpha>1$, $A_{n, \alpha}=n^{-1 / \alpha}\left(\sum_{i=1}^{n} a_{n, i}^{\alpha}\right)^{1 / \alpha}$. Except in Section 6 , where a simpler weighting will be considered, we will be interested in the convergence of $T_{n}=\sum_{i=1}^{n} a_{n, i} X_{i}$ assuming that, for some $\alpha>1$,

$$
A_{\alpha}=\sup _{n} A_{n, \alpha}<\infty
$$

This is a common assumption on the weighting coefficients (see, for example, Cuzick [3], Bai and Cheng [5] or Sung [6]). An extra assumption on the coefficients, allowing for the proof of a maximal inequality, will be used in Section 5, as was done in [8,9]. Observe that, due to the nonnegativity of the weights, the variables $T_{n}, n \geq 1$, are associated. 
Define the usual Cox-Grimmett coefficients

$$
u(n)=\sup _{k \geq 1} \sum_{j:|k-j| \geq n} \operatorname{Cov}\left(X_{j}, X_{k}\right)
$$

If the random variables are weakly stationary, then $u(n)=2 \sum_{j=n+1}^{\infty} \operatorname{Cov}\left(X_{1}, X_{j}\right)$. As moments and, especially, covariances will play a significant role throughout the paper, let us define, for each $i, j \geq 1$,

$$
\Delta_{i, j}(u, v)=\mathrm{P}\left(X_{i}>u, X_{j}>v\right)-\mathrm{P}\left(X_{i}>u\right) \mathrm{P}\left(X_{j}>v\right), \quad u, v \in \mathbb{R},
$$

and, for $x, y \geq 0$,

$$
G_{i, j}(x, y)=\iint_{[-x, x] \times[-y, y]} \Delta_{i, j}(u, v) d u d v
$$

It is obvious that $\operatorname{Cov}\left(X_{i}, X_{j}\right)=G_{i, j}(\infty, \infty)$. Moreover, observe that due to the association $\Delta_{i, j}$ is nonnegative, so it follows that each $G_{i, j}$ is nondecreasing in each variable.

Consider $p_{n}$ a sequence of natural numbers such that $p_{n}<\frac{n}{2}, r_{n}$ the largest integer less or equal to $\frac{n}{2 p_{n}}$, and define the variables

$$
Y_{n, j}=\sum_{i=(j-1) p_{n}+1}^{j p_{n}} a_{n, i} X_{i}, \quad j=1, \ldots, 2 r_{n}
$$

These random variables are associated, due to the nonnegativity of the weights. Note further that, if the variables $X_{n}$ are uniformly bounded by $c>0$, then it is obvious that $\left|Y_{n, j}\right| \leq c A_{\alpha} n^{1 / \alpha} p_{n}$. Finally, put

$$
T_{n, o d}=\sum_{j=1}^{r_{n}} Y_{n, 2 j-1} \quad \text { and } \quad T_{n, e v}=\sum_{j=1}^{r_{n}} Y_{n, 2 j} .
$$

We will need to describe the growth rate of sequences. For this, given sequences of nonnegative real numbers $a_{n}$ and $b_{n}$, we write $a_{n} \asymp b_{n}$ to mean that there exist constants $c_{1}>0$ and $c_{2}<\infty$ verifying $c_{1} \leq \frac{a_{n}}{b_{n}} \leq c_{2}$ for all $n$ large enough.

We prove first an easy but useful upper bound.

Lemma 2.1 Assume the variables $X_{n}, n \geq 1$, are centred, associated, weakly stationary, bounded (by $c>0$ ) and $u(0)<\infty$. Then $\mathrm{E} S_{n}^{2} \leq 2 c^{*} n$, where $c^{*}=c^{2}+u(0)$.

Proof. As the variables are stationary, it follows immediately that $\mathrm{E} S_{n}^{2}=n \operatorname{Var}\left(X_{1}\right)+$ $2 \sum_{j=1}^{n-1}(n-j) \operatorname{Cov}\left(X_{1}, X_{j+1}\right) \leq 2 n c^{2}+2 n u(0)$.

The next result is an extension of Lemma 3.1 in Oliveira [11].

Lemma 2.2 Assume the variables $X_{n}, n \geq 1$, are centred, associated, weakly stationary, bounded (by $c>0$ ), $u(0)<\infty$ and the nonnegative weights satisfy (1). If $d_{n}>1$ and 


$$
\begin{aligned}
& 0<\lambda<\frac{d_{n}-1}{d_{n}} \frac{1}{c A_{\alpha} n^{1 / \alpha} p_{n}} \text {, then } \\
& \qquad \max \left(\prod_{j=1}^{r_{n}} \mathrm{E} e^{\lambda Y_{n, 2 j-1}}, \prod_{j=1}^{r_{n}} \mathrm{E} e^{\lambda Y_{n, 2 j}}\right) \leq \exp \left(\lambda^{2} c^{*} A_{\alpha}^{2} n^{1+2 / \alpha} d_{n}\right) .
\end{aligned}
$$

Proof. We only prove the inequality for the product of even-indexed variables. As observed above, the variables $X_{n}$ being bounded, we have $\left|Y_{n, j}\right| \leq c A_{\alpha} n^{1 / \alpha} p_{n}$. So, using a Taylor expansion it follows that

$$
\mathrm{E} e^{\lambda Y_{n, 2 j-1}} \leq 1+\lambda^{2} \mathrm{E} Y_{n, 2 j-1}^{2} \sum_{k=2}^{\infty}\left(c A_{\alpha} \lambda n^{1 / \alpha} p_{n}\right)^{k-2} .
$$

Now, $\mathrm{E} Y_{n, 2 j-1}^{2}=\sum_{\ell, \ell^{\prime}=(j-1) p_{n}+1}^{j p_{n}} a_{n, \ell} a_{n, \ell^{\prime}} \operatorname{Cov}\left(X_{\ell}, X_{\ell^{\prime}}\right) \leq n^{2 / \alpha} A_{\alpha}^{2} \mathrm{E} S_{p_{n}}^{2}$, due to the stationarity and the nonnegativity of the weights and of the covariances. So, applying Lemma 2.1, it follows that

$$
\mathrm{E} e^{\lambda Y_{n, 2 j-1}} \leq 1+\frac{2 \lambda^{2} c^{*} A_{\alpha}^{2} n^{2 / \alpha} p_{n}}{1-c A_{\alpha} \lambda n^{1 / \alpha} p_{n}} \leq \exp \left(2 \lambda^{2} c^{*} A_{\alpha}^{2} n^{2 / \alpha} p_{n} d_{n}\right)
$$

To conclude the proof, multiply the upper bounds and remember that $2 r_{n} p_{n} \leq n$.

A basic tool for the analysis of the convergence and its rates is the following inequality, due to Dewan and Prakasa Rao [13].

Theorem 2.3 Assume $X_{1}, \ldots, X_{n}$ are centred, associated and uniformly bounded (by $c>0)$. Then, for every $\lambda>0$,

$$
\left|\mathrm{E} e^{\lambda \sum_{j=1}^{n} X_{j}}-\prod_{j=1}^{n} \mathrm{E} e^{\lambda X_{j}}\right| \leq \frac{1}{2} \lambda^{2} e^{c \lambda n} \sum_{\substack{j, k=1 \\ j \neq k}}^{n} \operatorname{Cov}\left(X_{j}, X_{k}\right) .
$$

\section{The case of uniformly bounded variables}

We assume first that there exists some $c>0$ such that, with probability $1,\left|X_{n}\right| \leq c$, for every $n \geq 1$. This allows for a direct use of the results in the previous section. We start by deriving an upper bound for the tail probabilities for $T_{n, o d}$ and $T_{n, e v}$.

Lemma 3.1 Assume the variables $X_{n}, n \geq 1$, are centred, associated, weakly stationary, bounded (by $c>0$ ) and $u(0)<\infty$. If the nonnegative weights satisfy (1), $d_{n}>1$ and $0<\lambda<\frac{d_{n}-1}{d_{n}} \frac{1}{c A_{\alpha} n^{1 / \alpha} p_{n}}$, then, for every $\varepsilon>0$ and $n$ large enough,

$$
\begin{gathered}
\mathrm{P}\left(T_{n, \text { od }}>n^{1 / p} \varepsilon\right) \leq \frac{1}{2} \lambda^{2} n^{1+2 / \alpha} A_{\alpha}^{2} \exp \left(\frac{1}{2} c n^{1+1 / \alpha} A_{\alpha} \lambda-\lambda n^{1 / p} \varepsilon\right) u\left(p_{n}\right) \\
+\exp \left(\lambda^{2} c^{*} A_{\alpha}^{2} n^{1+2 / \alpha} d_{n}-\lambda n^{1 / p} \varepsilon\right) .
\end{gathered}
$$

An analogous inequality for $\mathrm{P}\left(T_{n, e v}>n^{1 / p} \varepsilon\right)$ also holds. 
Proof. If we apply (4) to $T_{n, o d}$ we find

$$
\left|\mathrm{E} e^{\lambda T_{n, o d}}-\prod_{j=1}^{r_{n}} \mathrm{E} e^{\lambda Y_{n, 2 j-1}}\right| \leq \frac{1}{2} \lambda^{2} \exp \left(c A_{\alpha} r_{n} p_{n} n^{1 / \alpha} \lambda\right) \sum_{\substack{j, j^{\prime}=1 \\ j \neq j^{\prime}}}^{r_{n}} \operatorname{Cov}\left(Y_{n, j}, Y_{n, j^{\prime}}\right) .
$$

Now, as, for $n \geq 1$ and $i \leq n, 0 \leq a_{n, i} \leq n^{1 / \alpha} A_{n, \alpha}$, we have

$\operatorname{Cov}\left(Y_{n, j}, Y_{n, j^{\prime}}\right) \leq \sum_{\ell, \ell^{\prime}=(j-1) p_{n}+1}^{j p_{n}} a_{n, \ell} a_{n, \ell^{\prime}} \operatorname{Cov}\left(X_{\ell}, X_{\ell^{\prime}}\right) \leq n^{2 / \alpha} A_{\alpha}^{2} \sum_{\ell, \ell^{\prime}=(j-1) p_{n}+1}^{j p_{n}} \operatorname{Cov}\left(X_{\ell}, X_{\ell^{\prime}}\right)$

Put $Y_{n, j}^{*}=\sum_{\ell=(j-1) p_{n}+1}^{j p_{n}} X_{\ell}, j=1, \ldots, r_{n}$. Then, the previous inequality rewrites as

$$
\operatorname{Cov}\left(Y_{n, j}, Y_{n, j^{\prime}}\right) \leq n^{2 / \alpha} A_{\alpha}^{2} \operatorname{Cov}\left(Y_{n, j}^{*}, Y_{n, j^{\prime}}^{*}\right) .
$$

Using twice the stationarity of the random variables we obtain successively

$$
\sum_{\substack{j, j^{\prime}=1 \\ j \neq j^{\prime}}}^{r_{n}} \operatorname{Cov}\left(Y_{n, j}^{*}, Y_{n, j^{\prime}}^{*}\right)=2 \sum_{j=1}^{r_{n}-1}\left(r_{n}-j\right) \operatorname{Cov}\left(Y_{n, 1}^{*}, Y_{n, 2 j-1}^{*}\right)
$$

and

$$
\begin{aligned}
\operatorname{Cov}\left(Y_{n, 1}^{*}, Y_{n, 2 j-1}^{*}\right) & \leq \sum_{\ell=0}^{p_{n}-1}\left(p_{n}-\ell\right) \operatorname{Cov}\left(X_{1}, X_{2 j p_{n}+\ell+1}\right)+\sum_{\ell=1}^{p_{n}-1}\left(p_{n}-\ell\right) \operatorname{Cov}\left(X_{\ell}, X_{2 j p_{n}+1}\right) \\
& \leq p_{n} \sum_{\ell=(2 j-1) p_{n}+2}^{(2 j+1) p_{n}} \operatorname{Cov}\left(X_{1}, X_{\ell}\right) .
\end{aligned}
$$

Inserting these inequalities in (6), we find

$$
\begin{aligned}
\mid \mathrm{E} e^{\lambda T_{n, o d}}-\prod_{j=1}^{r_{n}} \mathrm{E} e^{\lambda Y_{n, 2 j-1} \mid} & \leq \lambda^{2} n^{2 / \alpha} A_{\alpha}^{2} r_{n} p_{n} \exp \left(\frac{1}{2} c n^{1+1 / \alpha} A_{\alpha} \lambda\right) \sum_{\ell=p_{n}+2}^{2\left(r_{n}-1\right) p_{n}} \operatorname{Cov}\left(X_{1}, X_{\ell}\right) \\
& \leq \frac{1}{2} \lambda^{2} n^{1+2 / \alpha} A_{\alpha}^{2} \exp \left(\frac{1}{2} c n^{1+1 / \alpha} A_{\alpha} \lambda\right) u\left(p_{n}+2\right) .
\end{aligned}
$$

We can now use this together with Markov's inequality to find that, for every $\varepsilon>0$,

$$
\begin{aligned}
\mathrm{P}\left(T_{n, o d}>n^{1 / p} \varepsilon\right) \leq & e^{-\lambda n^{1 / p} \varepsilon}\left|\mathrm{E} e^{\lambda T_{n, o d}}-\prod_{j=1}^{r_{n}} \mathrm{E} e^{\lambda Y_{n, 2 j-1}}\right|+e^{-\lambda n^{1 / p} \varepsilon} \prod_{j=1}^{r_{n}} \mathrm{E}^{\lambda Y_{n, 2 j-1}} \\
\leq & \frac{1}{2} \lambda^{2} n^{1+2 / \alpha} A_{\alpha}^{2} \exp \left(\frac{1}{2} c n^{1+1 / \alpha} A_{\alpha} \lambda-\lambda n^{1 / p} \varepsilon\right) u\left(p_{n}+2\right) \\
& +\exp \left(\lambda^{2} c^{*} A_{\alpha}^{2} n^{1+2 / \alpha} d_{n}-\lambda n^{1 / p} \varepsilon\right)
\end{aligned}
$$

and remember that $u\left(p_{n}+2\right) \leq u\left(p_{n}\right)$, due to the nonnegativity of the covariances. 
We may now prove the almost sure convergence of $n^{-1 / p} T_{n}$, assuming a convenient decrease rate on the Cox-Grimmett coefficients.

TheOREm 3.2 Assume the random variables $X_{n}, n \geq 1$, are centred, associated, strictly stationary and bounded (by $c>0$ ). Assume that $0<p<2$ and $\alpha>1$ are such that $\frac{1}{p}-\frac{1}{\alpha}=\frac{1}{2}+\xi$, for some $\xi>0$, and $u(n) \asymp \rho^{n}$, for some $\rho \in(0,1)$. If the nonnegative weights satisfy (1), then, with probability $1, n^{-1 / p} T_{n} \longrightarrow 0$.

Proof. Consider the decomposition of $T_{n}$ into the blocks $Y_{n, j}$ defined previously, taking $p_{n} \asymp n^{\theta}$, for some $\max \left(0, \frac{1}{2}-\xi\right)<\theta<\min \left(1, \frac{1}{2}+\xi\right)$. It is obviously enough to prove that both $n^{-1 / p} T_{n, o d}$ and $n^{-1 / p} T_{n, e v}$ converge almost surely to 0 . As these terms are analogous we will concentrate on the former, starting from (5). A minimization of the exponent on the second term of the upper bound in (5) leads to the choice

$$
\lambda=\frac{\varepsilon}{2 c^{*} A_{\alpha}^{2}} \frac{n^{1 / p-1-2 / \alpha}}{d_{n}},
$$

meaning that

$$
\exp \left(\lambda^{2} c^{*} A_{\alpha}^{2} n^{1+2 / \alpha} d_{n}-\lambda n^{1 / p} \varepsilon\right)=\exp \left(-\frac{\varepsilon^{2} n^{2 \xi}}{4 c^{*} A_{\alpha}^{2} d_{n}}\right) .
$$

Assume that, for some $\beta>1$,

$$
\frac{\varepsilon^{2} n^{2 \xi}}{4 c^{*} A_{\alpha}^{2} d_{n}}=\beta \log n \quad \Leftrightarrow \quad d_{n}=\frac{\varepsilon^{2}}{4 c^{*} A_{\alpha}^{2} \beta} \frac{n^{2 \xi}}{\log n} .
$$

As $\xi>0$, it follows that, for $n$ large enough, we have $d_{n}>1$ as required by Lemma 2.2. In order to use this lemma we also need to verify that the following condition on $\lambda$ is satisfied: $\lambda<\frac{d_{n}-1}{d_{n}} \frac{1}{c A_{\alpha} n^{1 / \alpha} p_{n}}$. Replacing (7) and remembering $d_{n}$ is larger than 1 , the assumption on Lemma 2.2 translates into

$$
\frac{\varepsilon}{2 c^{*} A_{\alpha}^{2}} \frac{n^{1 / p-1-2 / \alpha}}{d_{n}}<\frac{d_{n}-1}{d_{n}} \frac{1}{c A_{\alpha} n^{1 / \alpha} p_{n}}<\frac{1}{c A_{\alpha} n^{1 / \alpha} p_{n}} .
$$

Using now (8) to replace $d_{n}$, the previous inequality is equivalent to

$$
\frac{\varepsilon}{2 c^{*} A_{\alpha}^{2}} n^{1 / p-1-2 / \alpha}<\frac{1}{c A_{\alpha} n^{1 / \alpha+\theta}} \frac{\varepsilon^{2}}{4 c^{*} A_{\alpha}^{2} \beta} \frac{n^{2 \xi}}{\log n} \quad \Leftrightarrow \quad \varepsilon^{-1} \leq \frac{1}{2 c A_{\alpha} \beta} \frac{n^{1 / 2+\xi}}{n^{\theta} \log n} .
$$

As $\theta<\frac{1}{2}+\xi$ this upper bound grows to infinity, so this inequality is satisfied, at least for $n$ large enough.

We consider now the first term, involving the Cox-Grimmett coefficients, in (5). The exponent in this term is

$$
\frac{1}{2} c n^{1+1 / \alpha} A_{\alpha} \lambda-\lambda n^{1 / p} \varepsilon=\frac{c \varepsilon}{4 c^{*} A_{\alpha}} \frac{n^{1 / 2+\xi}}{d_{n}}-\frac{\varepsilon^{2}}{2 c^{*} A_{\alpha}^{2}} \frac{n^{2 \xi}}{d_{n}} .
$$

The second term above is, up to multiplication by 2 , the exponent that was found after the optimization with respect to $\lambda$ of the exponent on the second term of (5). So, to control 
the upper bound (5) we can factor this part of the exponential, leaving to control, after substitution the expressions for $\varepsilon$ and $d_{n}$,

$$
\frac{1}{4} \lambda^{2} n^{1+2 / \alpha} A_{\alpha}^{2} \exp \left(\frac{c \beta}{\varepsilon} n^{1 / 2-\xi} \log n\right) u\left(p_{n}\right) .
$$

As the term that we factored defines a convergent series, it is enough to verify that (10) is bounded. Further, the polynomial part in (10) is clearly dominated by the exponential, thus we may drop it, verifying only that there exists some $c_{0}>0$,

$$
\exp \left(\frac{c \beta}{\varepsilon} n^{1 / 2-\xi} \log n\right) u\left(p_{n}\right) \leq c_{0} \quad \Leftrightarrow \quad \frac{c \beta}{\varepsilon} n^{1 / 2-\xi} \log n+n^{\theta} \log \rho \leq \log c_{0} .
$$

But this is a consequence of $\theta>\frac{1}{2}-\xi$ and $\rho \in(0,1)$. Thus, given the above choices we have that $\mathrm{P}\left(\left|T_{n, o d}\right|>n^{1 / p} \varepsilon\right) \leq\left(c_{0}+1\right) n^{-\beta}$, where $\beta>1$, so $n^{-1 / p} T_{n, o d} \longrightarrow 0$ with probability 1 . Reasoning analogously we obtain the same result for $n^{-1 / p} T_{n, e v}$, so the proof is completed.

Remark 3.3 The decrease rate for $u(n)$ in Theorem 3.2 could be relaxed to a polynomial rate assuming that $\frac{1}{2}-\xi \leq 0$. But this implies that $\frac{1}{p}-\frac{1}{\alpha}>1$, hence that $p<1$. This, together with the boundedness assumption on the random variables makes the convergence proved trivial.

A small modification of the previous arguments allows for the identification of a convergence rate for the almost sure convergence just proved. To leave it clear about what we mean by a convergence rate in our framework, we will say that a sequence of random variables $Y_{n} \longrightarrow 0$ almost surely with rate $\varepsilon_{n} \searrow 0$, if $\varepsilon_{n}^{-1} Y_{n} \longrightarrow 0$ with probability 1 .

TheOREm 3.4 Assume the random variables $X_{n}, n \geq 1$, are centred, associated, strictly stationary and bounded (by $c>0$ ). Assume that $0<p<2$ and $\alpha>1$ are such that $\frac{1}{p}-\frac{1}{\alpha}=\frac{1}{2}+\xi$, for some $\xi>0$, and $u(n) \asymp \rho^{n}$, for some $\rho \in(0,1)$. If the nonnegative weights satisfy (1), then, with probability $1, n^{-1 / p} T_{n} \longrightarrow 0$ with convergence rate $\frac{\log n}{n^{\xi-\delta}}$, for arbitrarily small $\delta>0$.

Proof. We start again as in the proof of Theorem 3.2 by choosing $\theta=\frac{1}{2}+\delta$, with $0<\delta<\min \left(\frac{1}{2}, \xi\right)$ and $p_{n} \asymp n^{\theta}$. Now, on (8), allow $\varepsilon$ to depend on $n$ :

$$
\varepsilon_{n}^{2}=\frac{4 \beta c^{*} A_{\alpha}^{2} d_{n} \log n}{n^{2 \xi}}
$$

The verification of the assumptions of Lemma 2.2, given above by (9), becomes now:

$$
\frac{n^{\xi}}{2\left(\beta c^{*}\right)^{1 / 2} A_{\alpha} d_{n}^{1 / 2}(\log n)^{1 / 2}} \leq \frac{1}{2 c \beta A_{\alpha}} \frac{n^{\frac{1}{2}+\xi}}{n^{\frac{1}{2}+\delta} \log n},
$$

which is equivalent to $d_{n} \geq \frac{c^{2} \beta}{c^{*}} n^{2 \delta} \log n$. Thus, as we are interested in a slow growing sequence, we choose $d_{n} \asymp n^{2 \delta} \log n$. As a consequence, $\varepsilon_{n}^{2} \asymp n^{2(\delta-\xi)}(\log n)^{2} \longrightarrow 0$, given the choice for $\delta$. To complete the proof, it is enough to bound $\exp \left(\frac{1}{2} c n^{1+1 / \alpha} \lambda\right) u\left(p_{n}\right)$. It 
is easily verified that $n^{1+1 / \alpha} \lambda=\frac{1}{c} n^{1 / 2-\delta}$, so,

$$
\frac{1}{2} c n^{1+1 / \alpha} \lambda+\log u\left(p_{n}\right) \asymp n^{1 / 2-\delta}+n^{\theta} \log \rho=n^{1 / 2-\delta}+n^{1 / 2+\delta} \log \rho .
$$

But, the boundedness of this term is an immediate consequence of $\rho \in(0,1)$ and $\delta>0$, so the proof is concluded.

Example 3.5 Let $\varepsilon_{n}, n \in \mathbb{Z}$, be independent and identically distributed bounded and centred random variables with finite second order moment $\sigma^{2}$. Let $\rho \in(0,1)$, and define $X_{n}=\sum_{i=0}^{\infty} \rho^{i} \varepsilon_{n-i}$, which is an increasing transformation of the $\varepsilon_{n}$, so the variables $X_{n}$ are associated. Moreover, it is easy to verify that $\operatorname{Cov}\left(X_{n}, X_{m}\right)=\sigma^{2}\left(1-\rho^{2}\right)^{-1} \rho^{|n-m|}$, and the Cox-Grimmett coefficients are $u(n)=2 \sigma^{2}\left(1-\rho^{2}\right)^{-1}(1-\rho)^{-1} \rho^{n}$, hence satisfy the assumptions on Theorems 3.2 and 3.4. So, if we assume that the weights $a_{n, i}$ satisfy (1) for some $\alpha>1$, and $0<p<2$, it follows that $n^{-1 / p} \sum_{i=1}^{n} a_{n, i} X_{i} \longrightarrow 0$ almost surely. This application may also be addressed using Theorem 2.1 in Bai and Cheng [5] by rewriting the $X_{n}$ in terms of the independent random variables $\varepsilon_{n}$. These authors also assume (1) on the weight coefficients. However, after rewriting the variables, this means assuming that $\sup _{n} n^{-1} \sum_{i=1}^{n}\left(\sum_{j=i}^{\infty} \rho^{j} a_{n, i}\right)^{\alpha}<\infty$. If we take, for example, $a_{n, i}=n^{a} i^{-b}$, with $a<b$, it is easy to verify that this is strictly stronger than what follows from (1).

Example 3.6 As another application of the previous results consider now the weighted empirical distribution function

$$
H_{n}(x)=\frac{1}{n} \sum_{i=1}^{n} a_{n, i}\left(\mathbb{I}_{(-\infty, x]}\left(X_{i}\right)-F(x)\right),
$$

where $\mathbb{I}_{A}$ represents the characteristic function of the set $A$, the $X_{n}$ are associated and strictly stationary random variables with continuous distribution function $F$ with a bounded derivative, and the weights verify $\sum_{i=1}^{n} a_{n, i}=n$. Weighted empirical distribution functions appear in the literature when estimating the distribution function of a mixture of distributions (see, for example, Shcherbina [14]), or in goodness-of-fit problems as alternatives to the traditional empirical function (see, for instance, He et al. [15]). In order to apply Theorem 3.4, we need to control the Cox-Grimmett coefficients of the variables $\mathbb{I}_{(-\infty, x]}\left(X_{i}\right)$. It follows from Corollary 2.36 in [16] that there exists a constant $M$, depending only on the derivative of the distribution of the random variables, such that,

$$
\sum_{j=n}^{\infty} \operatorname{Cov}\left(\mathbb{I}_{(-\infty, x]}\left(X_{1}\right), \mathbb{I}_{(-\infty, x]}\left(X_{j}\right)\right) \leq M \sum_{j=n}^{\infty} \operatorname{Cov}^{1 / 3}\left(X_{1}, X_{j}\right)
$$

So, if $\operatorname{Cov}\left(X_{1}, X_{j}\right) \asymp \rho^{j}$, for some $\rho \in(0,1)$, the assumption in Theorem 3.4 on the Cox-Grimmett coefficients is satisfied.

The following result follows immediately from the discussion of the previous example.

COROllary 3.7 Let the random variables $X_{n}, n \geq 1$, are centred, associated and strictly stationary with distribution function $F$ with bounded derivative. Assume that $\alpha>2$, and $\operatorname{Cov}\left(X_{1}, X_{n}\right) \asymp \rho^{n}$, for some $\rho \in(0,1)$. If the nonnegative weights satisfy $\frac{1}{n} \sum_{i=1}^{n} a_{n, i} \longrightarrow$ 
1 and $\frac{1}{n} \sum_{i=1}^{n} a_{n, i}^{\alpha} \longrightarrow c^{\alpha}<\infty$, then, for each $x \in \mathbb{R}$, with probability 1 ,

$$
H_{n}^{*}(x)=\frac{1}{n} \sum_{i=1}^{n} a_{n, i} \mathbb{I}_{(-\infty, x]}\left(X_{i}\right) \longrightarrow F(x),
$$

with convergence rate $\frac{\log n}{n^{1 / 2-1 / \alpha-\delta}}$, for arbitrarily small $\delta>0$.

Proof. Define $p$ such that $\frac{1}{p}=\frac{1}{\alpha}+\frac{1}{2}+\xi$, for some $\xi>0$. The assumptions of Theorem 3.4 are satisfied so, a direct application of its conclusion gives that, with probability 1 ,

$$
\frac{n^{1+\xi-1 / p-\delta}}{\log n} H_{n} \longrightarrow 0 \text {. }
$$

As, $1+\xi-\frac{1}{p}-\delta=\frac{1}{2}-\frac{1}{\alpha}-\delta>0$, it follows that $H_{n} \longrightarrow 0$ almost surely. The definition of $p$ identifies the convergence rate immediately and the assumptions on the weights imply that, with probability 1 ,

$$
H_{n}(x)-H_{n}^{*}(x)=\frac{1}{n} \sum_{i=1}^{n} a_{n, i} F(x) \longrightarrow F(x) .
$$

Remark 3.8 Regarding the choice of weights in the previous corollary, one may try to define $a_{n, i}=n^{a} i^{b}$. The assumptions imply that $n^{a+b}$ and $n^{\alpha(a+b)}$ are asymptotically constant, hence are fulfillable if $a+b=0$.

A straightforward adaptation of Example 3.6 applies to nonparametric regression. Indeed, consider the model $Y_{n}=f\left(x_{n}\right)+\varepsilon_{n}, n \geq 1$, where the $x_{n}$ are fixed design points, $f$ an unknown regression function and the error variables $\varepsilon_{n}$ are centred, associated, strictly stationary and uniformly bounded. Define, for a given $x \in \mathbb{R}$, the estimator

$$
\widehat{f_{n}}(x)=\frac{1}{n} \sum_{i=1}^{n} a_{n, i}(x) Y_{i}
$$

COROLlary 3.9 Let $\varepsilon_{n}, n \geq 1$, be centred, associated, strictly stationary, and uniformly bounded random variables. Assume that $\alpha>2$ and $\operatorname{Cov}\left(\varepsilon_{1}, \varepsilon_{n}\right) \asymp \rho^{n}$, for some $\rho \in$ $(0,1)$, and $x_{n}, n \geq 1$, are fixed design points. For each $x \in \mathbb{R}$ define weights such that $\frac{1}{n} \sum_{i=1}^{n} a_{n, i}(x) f\left(x_{i}\right) \longrightarrow f(x)$. Then, with probability 1 , the estimator $\widehat{f}_{n}(x)$ converges to $f(x)$ with rate $\frac{\log n}{n^{1 / 2-1 / \alpha-\delta}}$, for arbitrarily small $\delta>0$.

\section{General random variables with infinitely many moments}

We begin by treating the case of general random variables assuming the existence of Laplace transforms in some neighbourhood of the origin. This is a strong assumption, as it implies the existence of moments of every order. However, it is in this case that we can fully extend the results proved in the previous section. Let us assume the random 
variables $X_{n}, n \geq 1$, are centred, strictly stationary and

$$
\exists M>0, \eta>0, \sup _{|t| \leq \eta} \mathrm{E} e^{t X_{1}} \leq M<\infty .
$$

For the present framework we cannot use directly Lemma 3.1, as this result depends on the boundedness of the variables. To circumvent this difficulty we introduce a truncation on the random variables, allowing to treat these truncated variables using the results in Section 3, and then control the remaining tails. Define, for each $c>0$, the nondecreasing functions $g_{c}(u)=\max (\min (u, c),-c), u \in \mathbb{R}$, which perform a truncation at level $c$. Let $c_{n}, n \geq 1$, be a sequence of nonnegative real numbers such that $c_{n} \longrightarrow \infty$ and define, for each $i, n \geq 1$,

$$
\begin{aligned}
& X_{1, i, n}=g_{c_{n}}\left(X_{i}\right)=-c_{n} \mathbb{I}_{\left(-\infty,-c_{n}\right)}\left(X_{i}\right)+X_{i} \mathbb{I}_{\left[-c_{n}, c_{n}\right]}\left(X_{i}\right)+c_{n} \mathbb{I}_{\left(c_{n}, \infty\right)}\left(X_{i}\right), \\
& X_{2, i, n}=\left(X_{i}-c_{n}\right) \mathbb{I}_{\left(c_{n}, \infty\right)}\left(X_{i}\right), \quad X_{3, i, n}=\left(X_{i}+c_{n}\right) \mathbb{I}_{\left(-\infty,-c_{n}\right)}\left(X_{i}\right) .
\end{aligned}
$$

Notice that the above transformations are monotonic, so these new families of variables are still associated. Moreover, it is obvious that, for each $n \geq 1$ fixed, the variables $X_{1,1, n}, \ldots, X_{1, n, n}$ are uniformly bounded. Consider, as before, an increasing sequence of natural numbers $p_{n}$ such that, for each $n \geq 1, p_{n}<\frac{n}{2}$ and define $r_{n}$ as the largest integer less than or equal to $\frac{n}{2 p_{n}}$. For $q=1,2,3$, and $j=1, \ldots, 2 r_{n}$, define

$$
Y_{q, j, n}=\sum_{i=(j-1) p_{n}+1}^{j p_{n}} a_{n, i}\left(X_{q, i, n}-\mathrm{E} X_{q, i, n}\right)
$$

and

$$
T_{q, n, o d}=\sum_{j=1}^{r_{n}} Y_{q, 2 j-1, n}, \quad T_{q, n, e v}=\sum_{j=1}^{r_{n}} Y_{q, 2 j, n} .
$$

For $q=2,3$, as we have assumed that the variables are strictly stationary, we find

$$
\begin{aligned}
\mathrm{P}\left(\left|\sum_{i=1}^{n} a_{n, i}\left(X_{q, i, n}-\mathrm{E} X_{q, i, n}\right)\right|>n^{1 / p} \varepsilon\right) & \leq n \mathrm{P}\left(\left|X_{q, 1, n}-\mathrm{E} X_{q, 1, n}\right|>\frac{n^{1 / p-1} \varepsilon}{A_{\alpha}}\right) \\
& \leq \frac{n^{3-2 / p} A_{\alpha}^{2}}{\varepsilon^{2}} \operatorname{Var}\left(X_{q, 1, n}\right) \leq \frac{n^{3-2 / p} A_{\alpha}^{2}}{\varepsilon^{2}} \mathrm{E} X_{q, 1, n}^{2}
\end{aligned}
$$

The following result is an easy extension of Lemma 4.1 in [11].

LEMMA 4.1 Let $X_{n}, n \geq 1$, be strictly stationary random variables satisfying (14). Then, for $t \in(0, \eta]$,

$$
\mathrm{P}\left(\left|\sum_{i=1}^{n} a_{n, i}\left(X_{q, i, n}-\mathrm{E} X_{q, i, n}\right)\right|>n^{1 / p} \varepsilon\right) \leq \frac{2 M A_{\alpha}^{2} n^{3-2 / p} e^{-t c_{n}}}{t^{2} \varepsilon^{2}}, q=2,3 .
$$

We may now prove the extensions of the results proved for uniformly bounded sequences of random variables. The main argument in the proofs in Section 3 was the control of the 
exponent in the exponential upper bounds found for the tail probabilities. The bound obtained in (18) is, essentially, of the same form, depending on the choice of the truncating sequence. So, we will obtain the same characterizations for the almost sure convergence and for its rate, as in the case of uniformly bounded sequences of random variables. Note that, due to the association of the variables,

$$
\operatorname{Cov}\left(X_{1,1, n}, X_{1, j, n}\right)=G_{1, j}\left(c_{n}, c_{n}\right) \leq G_{1, j}(\infty, \infty)=\operatorname{Cov}\left(X_{1}, X_{j}\right)
$$

Obviously, this inequality holds even if $\operatorname{Cov}\left(X_{1}, X_{j}\right)$ is not finite.

TheOREM 4.2 Assume the random variables $X_{n}, n \geq 1$, are centred, associated, strictly stationary and satisfy (14). Assume that $0<p<2$ satisfies $\frac{2}{p}>4-\eta$, and $\alpha>1$ are such that $\frac{1}{p}-\frac{1}{\alpha}=\frac{1}{2}+\xi$, for some $\xi>0$, and $u(n) \asymp \rho^{n}$, for some $\rho \in(0,1)$. If the nonnegative weights satisfy (1), then, with probability $1, n^{-1 / p} T_{n} \longrightarrow 0$.

Proof. To control the tail terms, that is, $T_{q, n, o d}$ and $T_{q, n, e v}$, for $q=2,3$, choose the truncating sequence $c_{n}=\log n$ and $t=\beta>4-\frac{2}{p}$. Thus, according to Lemma 4.1, the probabilities depending on these tail terms are bounded above by $n^{-\beta+3-2 / p}$, which defines a convergent series. Concerning the remaining term, we follow the proof of Theorem 3.2 keeping in mind that $c$ and $c^{*}$ now depend on $n$. Taking into account Lemma 2.1, we have $c_{n}^{*}=c_{n}^{2}+u(0) \asymp(\log n)^{2}$. Thus, instead of $(7)$, we find the choice

$$
\lambda=\frac{n^{1 / p-1-2 / \alpha} \varepsilon}{2 c_{n}^{*} A_{\alpha}^{2} d_{n}} \asymp \frac{n^{1 / p-1-2 / \alpha} \varepsilon}{d_{n}(\log n)^{2}},
$$

and

$$
\frac{n^{2 \xi} \varepsilon^{2}}{4 A_{\alpha}^{2} d_{n}(\log n)^{2}}=\beta \log n \quad \Leftrightarrow \quad d_{n}=\frac{\varepsilon^{2}}{4 \beta A_{\alpha}^{2}} \frac{n^{2 \xi}}{(\log n)^{3}}
$$

As $\xi>0$, this means that $d_{n}$ will, for $n$ large enough, be larger that 1 , as required by Lemma 2.2. To define the size of the blocks used to decompose the summations, choose $p_{n} \asymp n^{\theta}$, for some $\max \left(0, \frac{1}{2}-\xi\right)<\theta<\min \left(1, \frac{1}{2}+\xi\right)$. The condition on $\lambda$ required by Lemma 2.2 translates now into

$$
\varepsilon^{-1} \leq \frac{n^{1 / 2+\xi}}{2 c_{n} \beta A_{\alpha} n^{\theta} \log n} \asymp \frac{n^{1 / 2+\xi-\theta}}{(\log n)^{2}},
$$

thus, is verified, at least for $n$ large enough. We still have to control the behaviour of the term corresponding to (11). The exponent in this expression takes now the form $c_{n} n^{1+1 / \alpha} \lambda \asymp n^{1-1 / p+1 / \alpha}(\log n)^{2}$, that is, the same we found in course of proof of Theorem 3.2 multiplied by a logarithmic factor that, as is easily verified, does not affect the remaining argument of that proof.

Remark 4.3 Note that in Theorem 4.2 the lower bound $\frac{2}{p}>4-\eta$ is trivially satisfied whenever $\eta \geq 4$. Although a general interpretation seems difficult to find, it is possible to look at a few examples. Assume the random variables are distributed as $Y-\alpha \theta$ where $Y$ has distribution $\Gamma(\alpha, \theta)$ with density proportional to $x^{\alpha-1} e^{-x / \theta}$. Then, in (14) we may take $\eta=\frac{1}{\theta}$ and the assumption in Theorem 4.2 becomes trivial for $\theta<\frac{1}{4}$, that is, remembering that $\operatorname{Var}(Y)=\theta^{2} \alpha$, if the distribution of the random variable $Y-\alpha \theta$ is 
concentrated enough around its mean. The same characterization for the behaviour of the random variables holds if these follow a centred Gumbel distribution as the variance is now given by $\frac{\pi^{2} \theta^{2}}{6}$. Finally, to give an example with discrete distribution, assume the random variables behave now as a centred geometrically distributed variable with mean $\frac{1}{\theta}$, where $\theta \in[0,1]$. Referring to (14), we have now $\eta=-\log (1-p)$, hence our condition of Theorem 4.2 is trivially verified whenever $\theta>1-e^{-4}$, which corresponds to a geometric distribution with a rather low, smaller than $\frac{e^{-4}}{\left(1-e^{-4}\right)^{2}} \approx 0.019$, variance.

We state next the description of the convergence rate. The proof follows easily along the arguments used to prove Theorem 3.4 with adaptations similar to the ones used in the previous proof.

THEOREM 4.4 Assume the random variables $X_{n}, n \geq 1$, are centred, associated, strictly stationary and satisfy (14). Assume that $0<p<2$ satisfies $\frac{2}{p}>4-\eta$, and $\alpha>1$ are such that $\frac{1}{p}-\frac{1}{\alpha}=\frac{1}{2}+\xi$, for some $\xi>0$, and $u(n) \asymp \rho^{-n}$, for some $\rho \in(0,1)$. If the nonnegative weights satisfy (1), then, with probability $1, n^{-1 / p} T_{n} \longrightarrow 0$ with convergence rate $\frac{(\log n)^{2}}{n^{\xi-\delta}}$, for arbitrarily small $\delta>0$.

The above statements includes an assumption on the Cox-Grimmett coefficients of the original untruncated variables. In fact, this assumption may be relaxed, as we only need the coefficients corresponding to the truncated variables defined as, assuming already the stationarity of the variables,

$$
u^{*}(n)=2 \sum_{j=n+1}^{\infty} \operatorname{Cov}\left(X_{1,1, n}, X_{1, j, n}\right)=2 \sum_{j=n+1}^{\infty} G_{1, j}\left(c_{n}, c_{n}\right) \leq 2 \sum_{j=n+1}^{\infty} G_{1, j}(\infty, \infty)=u(n) .
$$

This allows for an immediate extension of Corollary 3.9 dropping the boundedness assumption on the errors. We state here the result, without proof as this is a straightforward adaptation of the arguments used before, as the final convergence rate is multiplied by a logarithmic factor.

COROLlaRY 4.5 Let $\varepsilon_{n}, n \geq 1$, be centred, associated and strictly stationary random variables and satisfy (14) with $\eta \geq 0$. Assume that $\alpha>2$ and $\operatorname{Cov}\left(\varepsilon_{1}, \varepsilon_{n}\right) \asymp \rho^{n}$, for some $\rho \in(0,1)$, and $x_{n}, n \geq 1$, are fixed design points. For each $x \in \mathbb{R}$ define weights such that $\frac{1}{n} \sum_{i=1}^{n} a_{n, i}(x) f\left(x_{i}\right) \longrightarrow f(x)$. Then, with probability 1 , the estimator $\widehat{f}_{n}(x)$ converges to $f(x)$ with rate $\frac{(\log n)^{2}}{n^{1 / 2-1 / \alpha-\delta}}$, for arbitrarily small $\delta>0$.

We discuss now a few examples, completing the analysis started in the previous section.

Example 4.6 Consider the linear process $Y_{n}=\sum_{i=0}^{n} a_{n, i} X_{n-i}$, where the variables $X_{n}$ are centred, strictly stationary and associated with $\operatorname{Cov}\left(X_{i}, X_{j}\right)=c \rho^{|j-i|}$, for some $\rho \in(0,1)$ and $c>0$. With respect to Example 3.5, we are dropping the boundedness assumption. The Cox-Grimmett coefficients for the variables $X_{n}$ are now given by $u(n)=$ $2 c(1-\rho)^{-1} \rho^{n}$. Assume the weights satisfy $a_{n, i}=n^{-a} i^{-b}$, for some $a, b>0$. We then have $Y_{n}=n^{-a} \sum_{i=0}^{n} i^{-b} X_{n-i}$ and (1) is obviously satisfied. It follows from Theorem 4.2 that $Y_{n} \longrightarrow 0$ almost surely if $a=\frac{1}{2}+\frac{1}{\alpha}+\xi$ where $\xi>0$ and $\alpha>1$, that is, if $a>\frac{1}{2}$.

This application improves the result proved after Corollary 2 in Louhichi [7]. Indeed, to derive the almost sure convergence $Y_{n} \longrightarrow 0$ for the same choice for the weights $a_{n, i}=$ $n^{-a} i^{-b}$, in [7] it is assumed that the random variables $X_{n}$ are independent, identically distributed, $a>\frac{1}{\alpha}+\frac{1}{2}$ and $b>\max \left(1,2+\frac{1}{\alpha}-\alpha a\right)$, hence require stronger conditions 
than those that follow from our Theorem 4.2.

Example 4.7 Consider again $Y_{n}=\sum_{i=0}^{n} a_{n, i} X_{n-i}$, where the variables $X_{n}$ are as in the previous example. Assume now the weights satisfy $a_{n, i}=n^{a} i^{-b}$ with $0<a<b$, so that (1) is fulfilled and consider $n^{-1 / p} Y_{n}=n^{-1 / p+a} \sum_{i=0}^{n} i^{-b} X_{n-i}$. The assumptions on the normalizing parameters $p$ and $\alpha$ above translate into $\frac{1}{p}-a-\frac{1}{\alpha}=\frac{1}{2}+\xi$, for some $\xi>0$, or, equivalently, $a<\frac{1}{p}-\frac{1}{2}$. So, if $0<a<\min \left(b, \frac{1}{p}-\frac{1}{2}\right)$, the linear process $n^{-1 / p} Y_{n}$ converges almost surely to 0 .

\section{General random variables with variance but only finitely many moments}

Assumption (14) used in the previous section is a rather strong one, as it implies the existence of every moment. Moreover, Lemma 4.1 does not use the dependence structure of the random variables to control the tail terms. In this section we will relax the assumptions on moments, thus requiring a different control on the tail terms. Instead of (14), we will assume a decrease rate on the tail joint probabilities:

$$
\sup _{i, j \geq 1} \Delta_{i, j}(x, y)=O\left(\max (|x|,|y|)^{-a}\right), \quad \text { for some } a>0, \text { as } \max (|x|,|y|) \longrightarrow \infty .
$$

Remark 5.1 It is easily verified that we have two different representations for the $\Delta_{i, j}$, each producing a different upper bound:

$$
\begin{aligned}
& \Delta_{i, j}(x, y)=\mathrm{P}\left(X_{i}>x, X_{j}>y\right)-\mathrm{P}\left(X_{i}>x\right) \mathrm{P}\left(X_{j}>y\right) \leq 2 \min \left(\mathrm{P}\left(X_{i}>x\right), \mathrm{P}\left(X_{j}>y\right)\right) \\
& \Delta_{i, j}(x, y)=\mathrm{P}\left(X_{i} \leq x, X_{j} \leq y\right)-\mathrm{P}\left(X_{i} \leq x\right) \mathrm{P}\left(X_{j} \leq y\right) \leq 2 \min \left(\mathrm{P}\left(X_{i} \leq x\right), \mathrm{P}\left(X_{j} \leq y\right)\right) .
\end{aligned}
$$

Thus, for $|x|$ or $|y|$ large enough, it follows that $\Delta_{i, j}(x, y) \leq 2 \min \left(\mathrm{P}\left(\left|X_{i}\right|>|x|\right), \mathrm{P}\left(\left|X_{j}\right|>\right.\right.$ $|y|))$. So, if we assume that $X_{n}, n \geq 1$, are identically distributed with tails such that $\mathrm{P}\left(\left|X_{1}\right|>x\right) \asymp x^{-a}$, as $x \longrightarrow \infty$, then the asymptotic behaviour described by (19) holds. As an example of a sequence fulfilling (19), consider $X_{n}, n \geq 1$, Pareto distributed and associated variables.

It is easily seen that the tail behaviour described in (19) only implies the existence of moments of order $k<a$. Besides, under (19), for $q=2,3$, and $i, j \geq 1$,

$$
\operatorname{Cov}\left(X_{q, i, n}, X_{q, j, n}\right) \leq c_{1} \iint_{\left[c_{n}, \infty\right)^{2}} \max (|x|,|y|)^{-a} d x d y=\frac{8 c_{1}}{a-2} c_{n}^{2-a},
$$

if $a>2$, where $c_{1}>0$ is a generic constant independent from $i, j$ and $n$.

The control of the tail terms will be achieved through a maximal inequality on weighted partial sums. Corresponding to the variables defined in (15), introduce the partial sums $T_{q, n}=\sum_{i=1}^{n} a_{n, i}\left(X_{q, i, n}-\mathrm{E} X_{q, i, n}\right), n \geq 1, q=1,2,3$. The following is an adapted version of Lemma 2.1 in Oliveira [8].

Lemma 5.2 Let $X_{n}, n \geq 1$, be centred and associated random variables. Assume the weights are such that

$$
a_{n, i} \geq 0, \quad \text { and } \quad a_{n, i} \geq a_{n-1, i}, \quad i<n, n \geq 1 .
$$


Then, for $q=1,2,3, \mathrm{E}\left(\max _{k \leq n} T_{q, k}^{2}\right) \leq \mathrm{E} T_{q, n}^{2}$.

Now that a maximal inequality is available, we may reproduce the arguments in the proof of Theorem 2 in Birkel [17] to prove a strong law of large numbers for the weighted sum. We include here the result without proof.

TheORem 5.3 Let $X_{n}, n \geq 1$, be centred and associated random variables. Assume that $0<p<2$ and $\alpha>1$ are such that $\frac{1}{p}-\frac{1}{\alpha}=\frac{1}{2}+\xi$, for some $\xi>0$. If the nonnegative weights satisfy (21) and

$$
\sum_{n=1}^{\infty} \frac{1}{n^{1+2 \xi}} \operatorname{Cov}\left(X_{n}, S_{n}\right)<\infty
$$

then, with probability $1, n^{-1 / p} T_{n} \longrightarrow 0$.

It is easily verified that if $\operatorname{Cov}\left(X_{n}, X_{m}\right) \asymp \rho^{|n-m|}$, for some $\rho \in(0,1)$, then $(22)$ is satisfied. Although Theorem 5.3 states an asymptotic result, it does not allow for the characterization of a convergence rate. For this purpose, we will use our previous approach based on exponential inequalities. We start by proving a simple upper bound for the tail probabilities of the weighted sums.

LEMmA 5.4 Let $X_{n}, n \geq 1$, be centred and associated random variables satisfying (19) with $a>2$. Assume $0<p<2$ and $\alpha>1$ are such that $\frac{1}{p}-\frac{1}{\alpha}=\frac{1}{2}+\xi$, for some $\xi>0$, and the weights satisfy (1) and (21). Then there exists a generic constant $c_{1}>0$ such that, for $q=2,3$,

$$
\mathrm{P}\left(\left|T_{q, n}\right|>n^{1 / p} \varepsilon\right) \leq \frac{8 c_{1}}{(a-2) \varepsilon^{2}} \frac{1}{n^{2 \xi-1} c_{n}^{a-2}}
$$

Proof. This is a straightforward consequence of Lemma 5.2, $a_{n, i} \leq n^{1 / \alpha} A_{\alpha}$ and (20).

We may now state sufficient conditions for the almost sure convergence of $n^{-1 / p} T_{n}$.

THEOREM 5.5 Assume the random variables $X_{n}, n \geq 1$, are centred, associated, strictly stationary satisfying (19) with $a>2$. Assume that $0<p<2$ and $\alpha>1$ are such that $\frac{1}{p}-\frac{1}{\alpha}=\frac{1}{2}+\xi$, for some $\xi \in\left(\frac{2}{a}, 1\right)$, and $u(n) \asymp \rho^{n}$, for some $\rho \in(0,1)$. If the nonnegative weights satisfy (1) and (21), then, with probability $1, n^{-1 / p} T_{n} \longrightarrow 0$.

Proof. Choose $c_{n}^{a-2}=n^{2-2 \xi}(\log n)^{b}$, for some $b>1$. As $\xi<1$, the truncating sequence $c_{n}$ does converge to $\infty$. Replacing this expression in (20), it follows that, for $q=2,3$,

$$
\sum_{n=1}^{\infty} \mathrm{P}\left(\left|T_{q, n}\right|>n^{1 / p} \varepsilon\right) \leq \frac{8 c_{1}}{(a-2) \varepsilon^{2}} \sum_{n=1}^{\infty} \frac{1}{n(\log n)^{b}}<\infty .
$$

Thus, it remains to prove that $\sum_{n} \mathrm{P}\left(\left|T_{1, n}\right|>n^{1 / p} \varepsilon\right)<\infty$. For this purpose, we will go along the arguments for the proof of Theorem 3.2. Choose $p_{n} \asymp n^{\theta}$ with $\frac{1}{2}-\xi+\frac{2-2 \xi}{a-2}<$ $\theta<\frac{1}{2}+\xi-\frac{2-2 \xi}{a-2}$ (it is easily verified that, as $a \xi>2$, this interval is nonempty). The minimization of the exponent in (5) leads to $\lambda=\frac{\varepsilon}{2 A_{\alpha}^{2}} \frac{n^{1 / p-1-2 / \alpha}}{c_{n}^{2} d_{n}}$, which gives raise to the term $\exp \left(-\frac{\varepsilon^{2} n^{2 \xi}}{4 A_{\alpha}^{2} c_{n}^{2} d_{n}}\right)$ on the upper bound. Thus, we will be interested in choosing the 
sequences such that, for some $\beta>1, \frac{\varepsilon^{2} n^{2 \xi}}{4 c_{n}^{2} A_{\alpha}^{2} d_{n}}=\beta \log n$, that is

$$
d_{n}=\frac{\varepsilon^{2}}{4 A_{\alpha}^{2} \beta} \frac{n^{2 \xi}}{c_{n}^{2} \log n}=\frac{\varepsilon^{2}}{4 A_{\alpha}^{2} \beta} n^{2 \xi-\frac{4(1-\xi)}{a-2}}(\log n)^{-(1+2 b /(a-2))}
$$

As $a \xi>2$ it follows that $\xi-2 \frac{1-\xi}{a-2}>0$, so $d_{n}$ chosen as above converges to $\infty$, becoming, for $n$ large enough, larger than 1 , as required by Lemma 2.2. This lemma also requires the verification of a condition on $\lambda$. Reasoning as in the proof of Theorem 3.2, this means that instead of (9) we need to verify that

$$
\varepsilon^{-1} \leq \frac{1}{2 A_{\alpha} \beta} \frac{n^{1 / 2+\xi}}{n^{\theta} c_{n} \log n}=\frac{1}{2 A_{\alpha} \beta} n^{\frac{1}{2}+\xi-\theta-\frac{2-2 \xi}{a-2}}(\log n)^{-(1+b /(a-2))} .
$$

As $\theta<\frac{1}{2}+\xi-\frac{2-2 \xi}{a-2}$ the exponent of $n$ in the previous expression is nonnegative, so this condition will be met, at least for $n$ large enough. To conclude the proof, we still have to bound the term

$$
\frac{1}{4} \lambda^{2} n^{1+2 / \alpha} A_{\alpha}^{2} \exp \left(\frac{\beta}{\varepsilon} c_{n} n^{1 / 2-\xi} \log n\right) u\left(p_{n}\right)
$$

where, as before, we may drop the polynomial term outside the exponential. After taking logarithms, the boundedness of this term is equivalent to finding an upper bound for

$$
c_{n} n^{1 / 2-\xi} \log n+n^{\theta} \log \rho=n^{\frac{1}{2}-\xi+\frac{2-2 \xi}{a-2}}(\log n)^{1+\frac{b}{a-2}}+n^{\theta} \log \rho .
$$

But, taking into account that $\rho \in(0,1)$ and the choice for $\theta$, the expression above is indeed bounded.

We may now adapt the arguments to characterize a convergence rate.

THEOREM 5.6 Assume the random variables $X_{n}, n \geq 1$, are centred, associated, strictly stationary satisfying (19) with $a>2$. Assume that $0<p<2$ and $\alpha>1$ are such that $\frac{1}{p}-\frac{1}{\alpha}=\frac{1}{2}+\xi$, for some $\xi>\frac{2}{a}$, and $u(n) \asymp \rho^{n}$, for some $\rho \in(0,1)$. If the nonnegative weights satisfy (1) and (21), then, with probability $1, n^{-1 / p} T_{n} \longrightarrow 0$, with convergence rate $\frac{(\log n)^{1+\eta_{1}}}{n^{\xi-2 / a-\eta_{2}}}$, for arbitrarily small $\eta_{1}, \eta_{2}>0$.

Proof. For the block decomposition choose $p_{n} \asymp n^{\theta}$, with $\theta=\frac{1}{2}+\delta$, where $0<\delta<$ $\min \left(\frac{1}{2}, \frac{a \xi-2}{a-2}\right)$. We will control the tail probabilities for $T_{q, n}$ using the arguments of Theorem 3.2 for $q=1$, and the previous theorem for $q=2$, 3 . For the case $q=1$, it follows that we want to choose

$$
\varepsilon_{n}^{2}=\frac{4 A_{\alpha}^{2} \beta c_{n}^{2} d_{n} \log n}{n^{2 \xi}}
$$

for some $\beta>1$, where $d_{n}>1$ and $c_{n} \longrightarrow \infty$. As in the proof of Theorem 3.4, we need to choose $d_{n}=\beta n^{2 \delta} \log n$ to fulfill the assumptions of Lemma 2.2. Now, to define the truncating sequence $c_{n}$ we use inequality (23), the representation for $\varepsilon_{n}$ above and the 
choice just made for $d_{n} \beta n^{2 \delta} \log n$, to find

$$
\begin{aligned}
\sum_{n=1}^{\infty} \mathrm{P}\left(\left|T_{1, n}\right|>n^{1 / p} \varepsilon\right) & \leq \frac{8 c_{1}}{(a-2)} \sum_{n=1}^{\infty} \frac{1}{\varepsilon_{n}^{2} n^{2 \xi-1} c_{n}^{a-2}}=\frac{8 c_{1}}{a-2} \sum_{n=1}^{\infty} \frac{n}{d_{n} c_{n}^{a} \log n} \\
& =\frac{8 c_{1}}{\beta(a-2)} \sum_{n=1}^{\infty} \frac{1}{n^{2 \delta-1} c_{n}^{a}(\log n)^{2}}
\end{aligned}
$$

Choose $c_{n}^{a}=n^{2(1-\delta)}(\log n)^{b}$, for some $b>0$, so the series above is convergent. With this choice we have

$$
\varepsilon_{n}^{2}=4 A_{\alpha}^{2} \beta^{2} \frac{(\log n)^{2+2 b / a}}{n^{2(\xi-\delta)-4(1-\delta) / a}}
$$

which identifies the convergence rate stated by taking $\eta_{1}=\frac{b}{a}$ and $\eta_{2}=\delta\left(1+\frac{2}{a}\right)$. To conclude we still need to control the term $\exp \left(\frac{A_{\alpha}}{2} c_{n} n^{1+1 / \alpha} \lambda\right) u\left(p_{n}\right)$. Now, $\frac{A_{\alpha}}{2} c_{n} n^{1+1 / \alpha} \lambda=$ $\frac{1}{2} n^{1 / 2-\delta}$, so

$$
\frac{A_{\alpha}}{2} c_{n} n^{1+1 / \alpha} \lambda+n^{1 / 2+\delta} \log \rho=\frac{1}{2} n^{1 / 2-\delta}+n^{1 / 2+\delta} \log \rho,
$$

is bounded as $\rho \in(0,1)$.

Çağın and Oliveira [9] prove in their Corollary 3.5 a result similar to our Theorems 4.2 and 5.5. The result in [9], besides always assuming the weights satisfy (1), (21) and $p \in(1,2)$, assumed

$$
\sum_{n=1}^{\infty} \int_{(n+1)^{(\alpha-2 p) /(\alpha p)}} v^{\frac{(\alpha-2) p}{\alpha-2 p}-3} G_{1, n}(v, v) d v<\infty .
$$

It is easily verified that if $\operatorname{Cov}\left(X_{1}, X_{n}\right)=\rho^{n}$, for some $\rho \in(0,1)$, the above assumption is satisfied whenever $\alpha>\frac{2 p}{2-p}$, but this is equivalent to $\frac{1}{p}-\frac{1}{\alpha}>\frac{1}{2}$. So, our Theorems 4.2 and 5.5 complement Corollary 3.5 in Çağın and Oliveira [9], strengthening the moment assumptions and enlarging the choice for the weights and the variability of $p$.

\section{A Marcinkiewicz-Zygmund law for random variables without means}

In the previous sections we considered stationary random variables with finite moments of order at least 2. We now drop the stationarity assumption and lower the requirement on moments to prove some Marcinkiewicz-Zygmund strong laws of large numbers. In this section, the method of approach is different, based on controlling moments of truncated variables, as done in Shen et al. [18], where these authors were interested in negatively associated random variables. As mentioned earlier, the dependence structure studied in [18] means that the control of variances of sums is easier than in the present framework, as these variances are smaller than the ones we find for sums of independent random variables. Thus, some extra care is required to control the moments in the sequel. This methodology does not allow for doubly indexed weights as in the previous sections, so we will obtain results with a somewhat more limited scope with respect to Oliveira [8] 
or Çağın and Oliveira [9]. However, we will complete a Marcinkiewicz-Zygmund strong law proved in Louhichi [7] by allowing normalizations of the form $n^{1 / p}$ with $p<1$ for random variables that do not have means. We start by recalling some general results on almost sure convergence. We recall that, unlike in the previous sections, we will not be assuming the stationarity of the random variables.

Theorem 6.1 (Theorem 3.1 in Prakasa Rao [19]) Let $X_{n}, n \geq 1$, be centred, square integrable and associated random variables. If $\sum_{i, j=1}^{\infty} \operatorname{Cov}\left(X_{i}, X_{j}\right)<\infty$ then $S_{n}$ is almost surely convergent (or, alternatively, $\sum_{n} X_{n}$ converges almost surely)

This result gives way to a version of the Three Series Theorem for associated sequences. Recall the nondecreasing functions $g_{c}(u)=\max (\min (u, c),-c), u \in \mathbb{R}$, where $c>0$ is fixed, and remember that if the original variables are associated then, as $g_{c}$ is nondecreasing, the sequence $g_{c}\left(X_{n}\right)$ is also associated.

Theorem 6.2 (Theorem 3.2 in Prakasa Rao [19]) Let $X_{n}, n \geq 1$, be associated random variables. Assume that for some $c>0$ we have

$$
\sum_{n=1}^{\infty} \mathrm{P}\left(\left|X_{n}\right|>c\right)<\infty, \quad \sum_{n=1}^{\infty} \mathrm{E} g_{c}\left(X_{n}\right)<\infty, \quad \sum_{i, j=1}^{\infty} \operatorname{Cov}\left(g_{c}\left(X_{i}\right), g_{c}\left(X_{j}\right)\right)<\infty
$$

Then $S_{n}$ converges almost surely (or, alternatively, $\sum_{n} X_{n}$ converges almost surely).

As for independent variables, the previous result enables the control of weighted sums of the form $\sum_{n} \frac{X_{n}}{a_{n}}$. We will assume the weights $a_{n}$ are, for every $n \geq 1$, nonnegative, so the quotients $\frac{X_{n}}{a_{n}}, n \geq 1$, are still associated. Introduce now a new sequence of associated random variables $Z_{n}=g_{1}\left(\frac{X_{n}}{a_{n}}\right), n \geq 1$. Note that the truncation at level 1 means no loss of generality, as truncation at any other level may be achieved replacing $a_{n}$ by $c a_{n}$. In order to prepare for the convergence result, we need some auxiliary inequalities.

LEMma 6.3 Let $h(\cdot)$ be an even function that is nondecreasing for $x>0$ and such that $\frac{x}{h(x)}$ is also nondecreasing for $x>0$. Then $\left|\mathrm{E} Z_{n}\right| \leq \mathrm{E}\left(\frac{h\left(X_{n}\right)}{h\left(a_{n}\right)}\right)$ and $\mathrm{E} Z_{n}^{2} \leq \mathrm{E}\left(\frac{h\left(X_{n}\right)}{h\left(a_{n}\right)}\right)$.

Proof. Write $\mathrm{E} Z_{n}=\mathrm{E}\left(\frac{X_{n}}{a_{n}} \mathbb{I}_{\left|X_{n}\right| \leq a_{n}}\right)+\mathrm{E}\left(\mathbb{I}_{\left|X_{n}\right|>a_{n}}\right)$. As $h$ is even and nondecreasing for $x>0, a_{n}<\left|X_{n}\right|$ implies $h\left(a_{n}\right) \leq h\left(X_{n}\right)$, thus $\mathrm{E}\left(\mathbb{I}_{\left|X_{n}\right|>a_{n}}\right) \leq \mathrm{E}\left(\frac{h\left(X_{n}\right)}{h\left(a_{n}\right)} \mathbb{I}_{\left|X_{n}\right|>a_{n}}\right)$. Now, as $\frac{x}{h(x)}$ is nondecreasing for $x>0$,

$$
\left|X_{n}\right| \leq a_{n} \quad \Rightarrow \quad \frac{\left|X_{n}\right|}{h\left(X_{n}\right)} \leq \frac{a_{n}}{h\left(a_{n}\right)} \quad \Leftrightarrow \quad \frac{h\left(a_{n}\right)}{h\left(X_{n}\right)} \leq \frac{a_{n}}{\left|X_{n}\right|} \quad \Leftrightarrow \quad \frac{\left|X_{n}\right|}{a_{n}} \leq \frac{h\left(X_{n}\right)}{h\left(a_{n}\right)} .
$$

Hence $\left|\mathrm{E}\left(\frac{X_{n}}{a_{n}} \mathbb{I}_{\left|X_{n}\right| \leq a_{n}}\right)\right| \leq \mathrm{E}\left(\frac{h\left(X_{n}\right)}{h\left(a_{n}\right)} \mathbb{I}_{\left|X_{n}\right| \leq a_{n}}\right)$, so summing the two upper bounds, the first inequality is proved. On what concerns the second inequality, write $\mathrm{E} Z_{n}^{2}=$ $\mathrm{E}\left(\frac{X_{n}^{2}}{a_{n}^{2}} \mathbb{I}_{\left|X_{n}\right| \leq a_{n}}\right)+\mathrm{E}\left(\mathbb{I}_{\left|X_{n}\right|>a_{n}}\right)$ and repeat the arguments above noting that, when $\left|X_{n}\right| \leq$ $a_{n}$ we have, as $h$ is nondecreasing for $x>0,0 \leq \frac{h\left(X_{n}\right)}{h\left(a_{n}\right)} \leq 1$, so $\frac{X_{n}^{2}}{a_{n}^{2}} \leq \frac{\left(h\left(X_{n}\right)\right)^{2}}{\left(h\left(a_{n}\right)\right)^{2}} \leq \frac{h\left(X_{n}\right)}{h\left(a_{n}\right)}$.

Lemma 6.4 Assume the same conditions as in Lemma 6.3. Then, for every $i \neq j$, $\left|\mathrm{E}\left(Z_{i} Z_{j}\right)\right| \leq \mathrm{E}\left(\frac{h\left(X_{i}\right)}{h\left(a_{i}\right)} \frac{h\left(X_{j}\right)}{h\left(a_{j}\right)}\right)$. 
Proof. Write the mathematical expectation as

$$
\begin{aligned}
\mathrm{E}\left(Z_{i} Z_{j}\right)=\mathrm{E}\left(\frac{X_{i}}{a_{i}} \frac{X_{j}}{a_{j}} \mathbb{I}_{\left|X_{i}\right| \leq a_{i}} \mathbb{I}_{\left|X_{j}\right| \leq a_{j}}\right)+\mathrm{E}\left(\frac{X_{i}}{a_{i}} \mathbb{I}_{\left|X_{i}\right| \leq a_{i}} \mathbb{I}_{\left|X_{j}\right|>a_{j}}\right) \\
+\mathrm{E}\left(\frac{X_{j}}{a_{j}} \mathbb{I}_{\left|X_{i}\right|>a_{i}} \mathbb{I}_{\left|X_{j}\right| \leq a_{j}}\right)+\mathrm{E}\left(\mathbb{I}_{\left|X_{i}\right|>a_{i}} \mathbb{I}_{\left|X_{j}\right|>a_{j}}\right)
\end{aligned}
$$

Reasoning as in the proof of Lemma 6.3 we can get the following inequalities:

$$
\begin{aligned}
& \left|\mathrm{E}\left(\frac{X_{i}}{a_{i}} \frac{X_{j}}{a_{j}} \mathbb{I}_{\left|X_{i}\right| \leq a_{i}} \mathbb{I}_{\left|X_{j}\right| \leq a_{j}}\right)\right| \leq \mathrm{E}\left(\frac{h\left(X_{i}\right)}{h\left(a_{i}\right)} \frac{h\left(X_{j}\right)}{h\left(a_{j}\right)} \mathbb{I}_{\left|X_{i}\right| \leq a_{i}} \mathbb{I}_{\left|X_{j}\right| \leq a_{j}}\right), \\
& \left|\mathrm{E}\left(\frac{X_{i}}{a_{i}} \mathbb{I}_{\left|X_{i}\right| \leq a_{i}} \mathbb{I}_{\left|X_{j}\right|>a_{j}}\right)\right| \leq \mathrm{E}\left(\frac{h\left(X_{i}\right)}{h\left(a_{i}\right)} \frac{h\left(X_{j}\right)}{h\left(a_{j}\right)} \mathbb{I}_{\left|X_{i}\right| \leq a_{i}} \mathbb{I}_{\left|X_{j}\right|>a_{j}}\right), \\
& \left|\mathrm{E}\left(\frac{X_{j}}{a_{j}} \mathbb{I}_{\left|X_{i}\right|>a_{i}} \mathbb{I}_{\left|X_{j}\right| \leq a_{j}}\right)\right| \leq \mathrm{E}\left(\frac{h\left(X_{i}\right)}{h\left(a_{i}\right)} \frac{h\left(X_{j}\right)}{h\left(a_{j}\right)} \mathbb{I}_{\left|X_{i}\right|>a_{i}} \mathbb{I}_{\left|X_{j}\right| \leq a_{j}}\right), \\
& \left|\mathrm{E}\left(\mathbb{I}_{\left|X_{i}\right|>a_{i}} \mathbb{I}_{\left|X_{j}\right|>a_{j}}\right)\right| \leq \mathrm{E}\left(\frac{h\left(X_{i}\right)}{h\left(a_{i}\right)} \frac{h\left(X_{j}\right)}{h\left(a_{j}\right)} \mathbb{I}_{\left|X_{i}\right|>a_{i}} \mathbb{I}_{\left|X_{j}\right|>a_{j}}\right) .
\end{aligned}
$$

Summing up these inequalities the conclusion of the lemma follows.

We may now state a general convergence result.

Theorem 6.5 Let $h(\cdot)$ be an even function that is nondecreasing for $x>0$ and such that $\frac{x}{h(x)}$ is also nondecreasing for $x>0$. Assume $X_{n}, n \geq 1$, are associated random variables such that

$$
\mathrm{E}\left(\sum_{n=1}^{\infty} \frac{h\left(X_{n}\right)}{h\left(a_{n}\right)}\right)^{2}<\infty
$$

Then $\sum_{n} \frac{X_{n}}{a_{n}}$ is almost surely convergent.

Proof. We will verify that the $Z_{n}$ random variables satisfy the assumptions of Theorem 6.2. As what regards the first assumption, using Markov's inequality,

$$
\sum_{n=1}^{\infty} \mathrm{P}\left(\left|Z_{n}\right|>1\right)=\sum_{n=1}^{\infty} \mathrm{P}\left(h\left(X_{n}\right)>h\left(a_{n}\right)\right) \leq \sum_{n=1}^{\infty} \frac{\mathrm{E}\left(h\left(X_{n}\right)\right)}{h\left(a_{n}\right)}=\mathrm{E}\left(\sum_{n=1}^{\infty} \frac{h\left(X_{n}\right)}{h\left(a_{n}\right)}\right)<\infty
$$

as the summation is assumed to be square integrable. The second summation in the assumptions of Theorem 6.2 is controlled applying twice the first upper bound proved in Lemma 6.3:

$$
\sum_{n=1}^{\infty}\left|\mathrm{E} Z_{n}\right| \leq 2 \sum_{n=1}^{\infty} \mathrm{E}\left(\frac{h\left(X_{n}\right)}{h\left(a_{n}\right)}\right)<\infty
$$


Finally,

$$
\begin{aligned}
\sum_{i, j=1}^{\infty} \operatorname{Cov}\left(Z_{i}, Z_{j}\right) & \leq \sum_{i, j=1}^{\infty}\left(\left|\mathrm{E}\left(Z_{i} Z_{j}\right)\right|+\left|\mathrm{E} Z_{i}\right|\left|\mathrm{E} Z_{j}\right|\right) \\
& \leq \sum_{i=1}^{\infty} \frac{\mathrm{E} h\left(X_{i}\right)}{h\left(a_{i}\right)}+\sum_{i \neq j} \mathrm{E}\left(\frac{h\left(X_{i}\right)}{h\left(a_{i}\right)} \frac{h\left(X_{j}\right)}{h\left(a_{j}\right)}\right)+\sum_{i, j=1}^{\infty} \frac{\mathrm{E} h\left(X_{i}\right)}{h\left(a_{i}\right)} \frac{\mathrm{E} h\left(X_{j}\right)}{h\left(a_{j}\right)} \\
& \leq \sum_{i=1}^{\infty} \frac{\mathrm{E} h\left(X_{i}\right)}{h\left(a_{i}\right)}+\mathrm{E}\left(\sum_{i=1}^{\infty} \frac{h\left(X_{i}\right)}{h\left(a_{i}\right)}\right)^{2}+\left(\sum_{i=1}^{\infty} \frac{\mathrm{E} h\left(X_{i}\right)}{h\left(a_{i}\right)}\right)^{2}<\infty
\end{aligned}
$$

Applying now Theorem 6.2, the conclusion follows.

COROLlary 6.6 Let $h(\cdot)$ be an even function that is nondecreasing for $x>0$ and such that $\frac{x}{h(x)}$ is also nondecreasing for $x>0$. Assume $X_{n}, n \geq 1$, are associated random variables such that (26) holds and the weights $a_{n} \nearrow \infty$. Then, $a_{n}^{-1} S_{n} \longrightarrow 0$ almost surely.

Proof. Apply Kronecker's Lemma to the conclusion of Theorem 6.5.

Example 6.7 Let $\varepsilon_{n}, n \in \mathbb{Z}$, be centred and associated random variables such that $\sup _{n \geq 1} \mathrm{E}\left|\varepsilon_{n}\right|^{2 q}<\infty$, for some $0<q \leq 1, \phi_{n}, n \geq 0$, be real numbers and define $X_{n}=\sum_{n=0}^{\infty} \phi_{i} \varepsilon_{n-i}$. Then, it follows from (26) applied to the $\varepsilon_{n}$ random variables and choosing $h(x)=|x|^{q}$, that $X_{n}$ is, with probability 1 , finite provided that $\sum_{i=0}^{\infty}\left|\phi_{i}\right|^{q}<\infty$. This is verified if, for example, $\phi_{n}=\rho^{n}$, for some $0<\rho<1$.

Moreover, to control moments of $X_{n}$, assume that $\frac{1}{2}<q \leq 1$, the $\phi_{n}$ are nonnegative, and rewrite $X_{n}=\sum_{n=0}^{\infty} \phi_{i}^{\gamma} \phi_{i}^{1-\gamma} \varepsilon_{n-i}$, for some $0<\gamma<1$. Applying Hölder inequality it follows $\mathrm{E}\left|X_{n}\right|^{2 q} \leq\left(\sum_{i=0}^{\infty} \phi_{i}^{2 q \gamma}\right)\left(\sum_{i=0}^{\infty} \phi_{i}^{2 q(1-\gamma) /(2 q-1)}\right)^{2 q-1} \mathrm{E}\left|\varepsilon_{n}\right|^{2 q}$, if both summations depending on the coefficients are finite. Thus, under the assumption $\sup _{n \geq 1} \mathrm{E}\left|\varepsilon_{n}\right|^{2 q}<\infty$, for some $\frac{1}{2}<q \leq 1$, it follows that $\sup _{n \geq 1} \mathrm{E}\left|X_{n}\right|^{2 q}<\infty$. So, applying again Theorem 6.5 now to the $X_{n}$ random variables, it follows that $\sum_{n=1}^{\infty} \frac{X_{n}}{a_{n}}$ is almost surely convergent if $\sum_{n=1}^{\infty} a_{n}^{-q}<\infty$.

We can find a condition for the convergence of $\sum_{i=1}^{n} \frac{X_{i}}{a_{i}}$ that is a somewhat weaker than (26) or, at least, may be written in a weaker form. It follows from the previous bounds that, for $i \neq j$,

$$
\operatorname{Cov}\left(Z_{i}, Z_{j}\right) \leq\left|\mathrm{E}\left(Z_{i} Z_{j}\right)\right|+\left|\mathrm{E} Z_{i}\right|\left|\mathrm{E} Z_{j}\right| \leq \operatorname{Cov}\left(\frac{h\left(X_{i}\right)}{h\left(a_{i}\right)}, \frac{h\left(X_{j}\right)}{h\left(a_{j}\right)}\right)+2 \frac{\mathrm{E} h\left(X_{i}\right)}{h\left(a_{i}\right)} \frac{\mathrm{E} h\left(X_{j}\right)}{h\left(a_{j}\right)} .
$$

Hence

$$
\sum_{i, j=1}^{\infty} \operatorname{Cov}\left(Z_{i}, Z_{j}\right) \leq \sum_{i=1}^{\infty} \frac{\mathrm{E} h\left(X_{i}\right)}{h\left(a_{i}\right)}+\sum_{i, j=1}^{\infty} \operatorname{Cov}\left(\frac{h\left(X_{i}\right)}{h\left(a_{i}\right)}, \frac{h\left(X_{j}\right)}{h\left(a_{j}\right)}\right)+2\left(\sum_{i=1}^{\infty} \frac{\mathrm{E} h\left(X_{i}\right)}{h\left(a_{i}\right)}\right)^{2} .
$$

We thus have the following alternative result. 
COROllary 6.8 Let $h(\cdot)$ be an even function that is nondecreasing for $x>0$ and such that $\frac{x}{h(x)}$ is also nondecreasing for $x>0$. Assume that $X_{n}, n \geq 1$, are associated random variables such that

$$
\sum_{i, j=1}^{\infty} \operatorname{Cov}\left(\frac{h\left(X_{i}\right)}{h\left(a_{i}\right)}, \frac{h\left(X_{j}\right)}{h\left(a_{j}\right)}\right)<\infty \quad \text { and } \quad \sum_{i=1}^{\infty} \frac{\mathrm{E} h\left(X_{i}\right)}{h\left(a_{i}\right)}<\infty .
$$

Then $\sum_{n} \frac{X_{n}}{a_{n}}$ is almost surely convergent. Moreover, if additionally the weights $a_{n} \nearrow \infty$, then $a_{n}^{-1} S_{n} \longrightarrow 0$ almost surely.

We may now prove first a result for general weights with a control on moments of the random variables.

TheOREM 6.9 Let $X_{n}, n \geq 1$, be associated random variables and $0<q \leq 1$ be such that $\sup _{n \geq 1} \mathrm{E}\left|X_{n}\right|^{q}<\infty$. Assume the positive weights $a_{n}$ are such that

$$
\sum_{n=1}^{\infty} \frac{1}{a_{n}^{q}}<\infty \quad \text { and } \quad \sum_{i=1}^{\infty} \sum_{j=i+1}^{\infty} \frac{1}{a_{i} a_{j}} G_{i, j}\left(a_{i}, a_{j}\right)<\infty .
$$

Then $\sum_{n} \frac{X_{n}}{a_{n}}$ is almost surely convergent. Moreover, if additionally the weights $a_{n} \nearrow \infty$, then $a_{n}^{-1} S_{n} \longrightarrow 0$ almost surely.

Proof. Choose $h(x)=|x|^{q}$, fulfilling the assumptions on $h$ of the preceding results. Then, the second summation in (27) is bounded above by

$$
\sup _{n \geq 1} \mathrm{E}\left|X_{n}\right|^{q} \sum_{n=1}^{\infty} \frac{1}{a_{n}^{q}}<\infty .
$$

As what regards the second inequality in (27), we go back to $\operatorname{Cov}\left(Z_{i}, Z_{j}\right)$. We may write, using the $\Delta_{i, j}$ functions defined in (3),

$$
\begin{aligned}
& \operatorname{Cov}\left(Z_{i}, Z_{j}\right)=\iint_{[-1,1]^{2}} \Delta_{i, j}\left(a_{i} u, a_{j} v\right) d u d v \\
& \quad=\frac{1}{a_{i} a_{j}} \iint_{\left[-a_{i}, a_{i}\right] \times\left[-a_{j}, a_{j}\right]} \Delta_{i, j}(u, v) d u d v=\frac{1}{a_{i} a_{j}} G_{i, j}\left(a_{i}, a_{j}\right) .
\end{aligned}
$$

Finally, using the second inequality from Lemma 6.3,

$$
\begin{aligned}
\sum_{i, j=1}^{\infty} \operatorname{Cov}\left(Z_{i}, Z_{j}\right) & =\sum_{i=1}^{\infty} \operatorname{Var}\left(Z_{i}\right)+2 \sum_{i<j} \frac{1}{a_{i} a_{j}} G_{i, j}\left(a_{i}, a_{j}\right) \\
& \leq \sum_{i=1}^{\infty} \mathrm{E} Z_{i}^{2}+2 \sum_{i<j} \frac{1}{a_{i} a_{j}} G_{i, j}\left(a_{i}, a_{j}\right) \\
& \leq \mathrm{E}\left|X_{1}\right|^{q} \sum_{i=1}^{\infty} \frac{1}{a_{i}^{q}}+2 \sum_{i<j} \frac{1}{a_{i} a_{j}} G_{i, j}\left(a_{i}, a_{j}\right)<\infty
\end{aligned}
$$

so the proof is concluded. 
Example 6.10 Assume again that $X_{n}=\sum_{i=0}^{\infty} \phi_{i} \varepsilon_{n-i}$, where the $\varepsilon_{k}, k \in \mathbb{Z}$, are associated and now stationary random variables such that $\mathrm{E}\left|\varepsilon_{1}\right|^{q}<\infty$, for some $0<q \leq 1$, and $\phi_{n} \geq 0$. Note that, reproducing the arguments in the second part of Example 6.7, we have, assuming the convergence of the series depending on the coefficients mentioned there, $\sup _{n>1} \mathrm{E}\left|X_{n}\right|^{q}<\infty$. For the control of the truncated covariances, recall again that $g_{c}(u)=\max (\min (u, c),-c)$, and $G_{i, j}(u, v)=\operatorname{Cov}\left(g_{u}\left(X_{i}\right), g_{v}\left(X_{j}\right)\right)$, so, for any given $0<\gamma<1$ and $u, v>0$,

$$
\begin{aligned}
G_{i, j}(u, v) & =\mathrm{E} g_{u}\left(X_{i}\right) g_{v}\left(X_{j}\right)-\mathrm{E} g_{u}\left(X_{i}\right) \mathrm{E} g_{v}\left(X_{j}\right) \\
& \leq \mathrm{E}\left(\min \left(u,\left|X_{i}\right|\right) \min \left(v,\left|X_{j}\right|\right)\right)+\mathrm{E} \min \left(u,\left|X_{i}\right|\right) \mathrm{E} \min \left(v,\left|X_{j}\right|\right) \\
& \leq u^{\gamma} v^{\gamma}\left(\mathrm{E}\left(\left|X_{i}\right|^{1-\gamma}\left|X_{j}\right|^{1-\gamma}\right)+\mathrm{E}\left|X_{i}\right|^{1-\gamma} \mathrm{E}\left|X_{j}\right|^{1-\gamma}\right) \\
& \leq 2 u^{\gamma} v^{\gamma} \mathrm{E}\left|X_{1}\right|^{2(1-\gamma)},
\end{aligned}
$$

if we choose $1-\gamma<\frac{q}{2}$, using the Hölder inequality and taking into account that the $X_{n}$, $n \geq 1$, are stationary. Hence, concerning the second condition in (28), we have that

$$
\sum_{i=1}^{\infty} \sum_{j=i+1}^{\infty} \frac{1}{a_{i} a_{j}} G_{i, j}\left(a_{i}, a_{j}\right) \leq 2 \sum_{i=1}^{\infty} \sum_{j=i+1}^{\infty} \frac{1}{a_{i}^{1-\gamma} a_{j}^{1-\gamma}} \leq\left(\sum_{i=1}^{\infty} \frac{1}{a_{i}^{1-\gamma}}\right)^{2} .
$$

So, if this last summation is finite, as $q>2(1-\gamma)$, the first condition in (28) is also fulfilled and, taking into account Theorem $6.9, \sum_{n} \frac{X_{n}}{a_{n}}$ converges almost surely.

Note that using the same exponent $\gamma$ above is just convenient for the explicit calculation of the series. So, choosing different $\gamma_{i}$ 's, the above construction justifies that one can find models for which $G_{i, j}(u, v)$ is controlled by $u^{\gamma_{i}} v^{\gamma_{j}}$.

Example 6.11 For $q=1$ we should compare the second conclusion of Theorem 6.9 with Theorem 1 in Louhichi [7]. This later result from [7] assumes the association of the random variables, the existence of an integrable random variable $X$ stochastically dominating the $X_{n}, n \geq 1$, that is such that, for every $n \geq 1, \mathrm{P}\left(\left|X_{n}\right|>x\right) \leq \mathrm{P}(|X|>x)$, and uses a weaker normalizing sequence: $n$ instead of $a_{n}=n^{1+\eta}$, with $\eta>0$, as required by our Theorem 6.9. Louhichi's [7] result requires further an extra condition on the growth rate of the truncated covariances:

$$
\sum_{i=1}^{\infty} \sum_{j=i+1}^{\infty} \int_{j^{1 / q}}^{\infty} \frac{1}{v^{3}} G_{i, j}(v, v) d v<\infty
$$

This is similar to the second condition in our assumption (28). However, our version seems somewhat less restrictive as shown by the following example: assume, as referred above, that $a_{n}=n^{1+\eta}$, for some $\eta>0$, and $G_{i, j}(x, y)=x^{\alpha} y^{\beta}$, for suitable $\alpha, \beta>0$, thus not depending on both indices $i$ and $j$. In this case, the summation in (29) diverges, as the terms will be constant with respect to the first summation index $i$. On what regards the second assumption in (28), we have

$$
\sum_{i=1}^{\infty} \sum_{j=i+1}^{\infty} \frac{1}{a_{i} a_{j}} G_{i, j}\left(a_{i}, a_{j}\right)=\sum_{i=1}^{\infty} i^{(\alpha-1)(1+\eta)} \sum_{j=i+1}^{\infty} j^{(\beta-1)(1+\eta)} \asymp \sum_{i=1}^{\infty} i^{1+(\alpha+\beta-2)(1+\eta)}<\infty,
$$

provided that $\alpha+\beta<\frac{2 \eta}{1+\eta}$. Thus, the assumptions of Theorem 6.9 are satisfied while 
Theorem 1 in Louhichi [7] is not applicable.

Example 6.12 For $0<q<1$, Chandra and Ghosal [12], in their Remark 3, note that $n^{-1 / q} S_{n} \longrightarrow 0$ almost surely provided there exists a random variable $X$ stochastically dominating the $X_{n}, n \geq 1$, and $\mathrm{E}|X|^{q}<\infty$. Thus, compared with Theorem 6.9, no dependence assumption is required. Moreover, the normalizing sequence in Chandra and Ghosal's [12] result is strictly weaker, as the first inequality in (28) implies that the normalizing sequence $a_{n}$ in Theorem 6.9 is strictly larger than $n^{1 / q}$. Thus our result is, in this respect, weaker than what is proved in Chandra and Ghosal [12]. On the other hand, Theorem 6.9 replaces the stochastic domination by the association assumption, thus offering some relaxation in this direction. An example where Theorem 6.9 provides convergence but the conditions of Chandra and Ghosal [12] are not necessarily fulfilled is obtained in a similar way as in our previous example: choose the normalizing sequence $a_{n}=n^{1 / p}$ and $G_{i, j}(x, y)=x^{\alpha_{i}} y^{\beta_{j}}$, where $0<\alpha_{i}, \beta_{j}<1-p$, at least for large $i$ or $j$. Then, it is easy to verify that both conditions in (28) are satisfied, thus all that is required to have the almost sure convergence is $\sup _{n \geq 1} \mathrm{E}\left|X_{n}\right|^{q}<\infty$, where $p<q \leq 1$, which is weaker than the stochastic dominance required in Chandra and Ghosal's [12] result.

Remark 6.13 Concerning the normalizing sequence, Theorem 6.9 will always require a normalizing sequence strictly stronger than the optimal one for Marcinkiewciz-Zygmund laws. Indeed, when normalizing by $n^{1 / p}$ we will have to require that $\sup _{n \geq 1} \mathrm{E}\left|X_{n}\right|^{q}<\infty$ for some $q>p$, as the same function $h$ appears in both the numerator and denominator of the first condition in (28). Of course, to derive that $n^{-1 / p} S_{n} \longrightarrow 0$ almost surely we still would need to assume that

$$
\sum_{i=1}^{\infty} \sum_{j=i+1}^{\infty} \frac{1}{i^{1 / p} j^{1 / p}} G_{i, j}\left(i^{1 / p}, j^{1 / p}\right)<\infty .
$$

Remark 6.14 The verification of (30) may be achieved by assuming a decrease rate on the joint tail probabilities (3). In fact, assume that (19) holds for some $a>2 p$. It follows that there exist $u_{0}>0$ and $c_{1}>0$ such that for $|u|,|v| \geq u_{0}$ and every $i, j \geq 1$ we have $\Delta_{i, j}(u, v) \leq c_{1} \max (|u|,|v|)^{-a}$. Remember that, as mentioned in Remark 5.1, this holds if the tail of the distribution of the variables decreases polynomially with exponent $a$. As, obviously, for every $u, v \in \mathbb{R}$ and $i, j \geq 1, \Delta_{i, j}(u, v) \leq 1$, we have, for $i<j$,

$$
\begin{aligned}
G_{i, j}\left(i^{1 / p}, j^{1 / p}\right) \leq & u_{0}^{2} \\
+ & 2 c_{1} \int_{u_{0}}^{i^{1 / p}} \int_{-u}^{u} d v|u|^{-a} d u+2 c_{1} \int_{u_{0}}^{i^{1 / p}} \int_{-v}^{v} d u|v|^{-a} d v \\
& \quad+2 c_{1} \int_{i^{1 / p}}^{j^{1 / p}} \int_{-i^{1 / p}}^{i^{1 / p}} d u v^{-a} d v \\
= & \left(u_{0}^{2}-\frac{8 c_{1}}{2-a} u_{0}^{2-a}\right)-\frac{4 c_{1}}{(2-a)(1-a)} i^{(2-a) / p}+\frac{4 c_{1}}{1-a} i^{1 / p} j^{(1-a) / p} .
\end{aligned}
$$

Replacing this in (30) and dropping the constants, we are left with three series to control. For one corresponding to the first term in (31) we have, as $p<1$,

$$
\sum_{i=1}^{\infty} \sum_{j=i+1}^{\infty} i^{-1 / p} j^{-1 / p} \leq \frac{1}{2}\left(\sum_{i=1}^{\infty} i^{-1 / p}\right)^{2}<\infty .
$$


The series corresponding to the second term in (31), taking into account that $a>2 p$ implies $1-\frac{a}{p}<-1$, is

$$
\begin{aligned}
\sum_{i=1}^{\infty} \sum_{j=i+1}^{\infty} i^{(2-a) / p} i^{-1 / p} j^{-1 / p}=\sum_{i=1}^{\infty} i^{(1-a) / p} \sum_{j=i+1}^{\infty} j^{-1 / p} \\
\asymp \sum_{i=1}^{\infty} i^{(1-a) / p} i^{1-1 / p}=\sum_{i=1}^{\infty} i^{1-a / p}<\infty
\end{aligned}
$$

Finally, using again that $1-\frac{a}{p}<-1$, the third term in (31) is

$$
\sum_{i=1}^{\infty} \sum_{j=i+1}^{\infty} i^{-1 / p} j^{-1 / p} i^{1 / p} j^{(1-a) / p}=\sum_{i=1}^{\infty} \sum_{j=i+1}^{\infty} j^{-a / p}<\sum_{j=2}^{\infty} \sum_{i=1}^{j} j^{-a / p}=\sum_{j=2}^{\infty} j^{1-a / p}<\infty
$$

\section{Acknowledgement}

The authors wish to thank the anonymous referee and the Associate Editor whose careful reading and suggestions helped improving on an earlier versions of this paper.

\section{References}

[1] Baum L, Katz M. Convergence rates in the law of large numbers. Bull. Amer. Math. Soc. 1963;69: 771772.

[2] Chow Y. Some convergence theorems for independent random variables. Ann. Math. Statist. 1966;37:1482-1493.

[3] Cuzick J. A Strong Law for Weighted Sums of I.I.D. Random Variables. J. Theoret. Probab. 1995;8:625-641.

[4] Cheng P. A note on strong convergence rates in nonparametric regression. Statist. Probab. Lett. 1995;24:357-364.

[5] Bai Z, Cheng P. Marcinkiewicz strong laws for linear statistics. Statist. Probab. Lett. 2000;46:105-112.

[6] Sung S. Strong laws for weighted sums of i.i.d. random variables. Statist. Probab. Lett. 2001;52:413-419.

[7] Louhichi S. Convergence rates in the strong law for associated random variables. Probab. Math. Statist. 2000;20:203-214.

[8] Oliveira PE. Weighted sums of associated variables. J. Korean Statist. Soc. 2012;41:537-542.

[9] Çağın T, Oliveira PE. A Note on Weighted Sums of Associated Random Variables. Acta Math. Hungar. 2014;143:pp. 96-106.

[10] Ioannides DA, Roussas GG. Exponential inequality for associated random variables. Statist. Probab. Lett. 1999;42:pp. 423-431.

[11] Oliveira PE. An exponential inequality for associated variables. Statist. Probab. Lett. 2005;73:189-197.

[12] Chandra TK, Ghosal, SH. Extensions of the strong law of large numbers of Marcinkiewicz and Zygmund for dependent variables. Acta Math. Hungar. 1996;71:327-336.

[13] Dewan I, Prakasa Rao BLS. A general method of density estimation for associated random variables. J. Nonparametr. Statist. 1999;10:405-420.

[14] Shcherbina, A. Estimation of the mean value in a model of mixtures with varying concentrations. Theor. Probability and Math. Statist. 2012;84:151-164. 

$\begin{aligned} & \\ & {[15] } \text { He D, Xu K, Xu X. Random Weighting Empirical Distribution Function and its Applications } \\ & \text { to Goodness-of-Fit Testing. Comm. Statist. Simulation Comput. 2015;44:1441-1452. } \\ & {[16] } \text { Oliveira PE. Asymptotics for Associated Random Variables. Springer;2012. } \\ & {[17] } \text { Birkel, T. A note on the strong law of large numbers for positively dependent random } \\ & \text { variables. Statist. Probab. Lett. 1989;7:17-20. } \\ & {[18] } \text { Shen Y, Wang X, Yang W, Hu S. Almost sure convergence theorem and strong stabi- } \\ & \text { lity for weighted sums of NSD random variables. Acta Mathematica Sinica, English Series } \\ & 2013 ; 29: 743-756 . \\ & {[19] } \text { Prakasa Rao, BLS. Hajek-Renyi-type inequality for associated sequences. Statist. Probab. } \\ & \text { Lett. 2002;57:139-143 }\end{aligned}$ $\begin{aligned} & \\ & {[15] } \text { He D, Xu K, Xu X. Random Weighting Empirical Distribution Function and its Applications } \\ & \text { to Goodness-of-Fit Testing. Comm. Statist. Simulation Comput. 2015;44:1441-1452. } \\ & {[16] } \text { Oliveira PE. Asymptotics for Associated Random Variables. Springer;2012. } \\ & {[17] } \text { Birkel, T. A note on the strong law of large numbers for positively dependent random } \\ & \text { variables. Statist. Probab. Lett. 1989;7:17-20. } \\ & {[18] } \text { Shen Y, Wang X, Yang W, Hu S. Almost sure convergence theorem and strong stabi- } \\ & \text { lity for weighted sums of NSD random variables. Acta Mathematica Sinica, English Series } \\ & 2013 ; 29: 743-756 . \\ & {[19] } \text { Prakasa Rao, BLS. Hajek-Renyi-type inequality for associated sequences. Statist. Probab. } \\ & \text { Lett. 2002;57:139-143 }\end{aligned}$ $\begin{aligned} & \\ & {[15] } \text { He D, Xu K, Xu X. Random Weighting Empirical Distribution Function and its Applications } \\ & \text { to Goodness-of-Fit Testing. Comm. Statist. Simulation Comput. 2015;44:1441-1452. } \\ & {[16] } \text { Oliveira PE. Asymptotics for Associated Random Variables. Springer;2012. } \\ & {[17] } \text { Birkel, T. A note on the strong law of large numbers for positively dependent random } \\ & \text { variables. Statist. Probab. Lett. 1989;7:17-20. } \\ & {[18] } \text { Shen Y, Wang X, Yang W, Hu S. Almost sure convergence theorem and strong stabi- } \\ & \text { lity for weighted sums of NSD random variables. Acta Mathematica Sinica, English Series } \\ & 2013 ; 29: 743-756 . \\ & {[19] } \text { Prakasa Rao, BLS. Hajek-Renyi-type inequality for associated sequences. Statist. Probab. } \\ & \text { Lett. 2002;57:139-143 }\end{aligned}$ $\begin{aligned} & \\ & {[15] } \text { He D, Xu K, Xu X. Random Weighting Empirical Distribution Function and its Applications } \\ & \text { to Goodness-of-Fit Testing. Comm. Statist. Simulation Comput. 2015;44:1441-1452. } \\ & {[16] } \text { Oliveira PE. Asymptotics for Associated Random Variables. Springer;2012. } \\ & {[17] } \text { Birkel, T. A note on the strong law of large numbers for positively dependent random } \\ & \text { variables. Statist. Probab. Lett. 1989;7:17-20. } \\ & {[18] } \text { Shen Y, Wang X, Yang W, Hu S. Almost sure convergence theorem and strong stabi- } \\ & \text { lity for weighted sums of NSD random variables. Acta Mathematica Sinica, English Series } \\ & 2013 ; 29: 743-756 . \\ & {[19] } \text { Prakasa Rao, BLS. Hajek-Renyi-type inequality for associated sequences. Statist. Probab. } \\ & \text { Lett. 2002;57:139-143 }\end{aligned}$ $\begin{aligned} & \\ & {[15] } \text { He D, Xu K, Xu X. Random Weighting Empirical Distribution Function and its Applications } \\ & \text { to Goodness-of-Fit Testing. Comm. Statist. Simulation Comput. 2015;44:1441-1452. } \\ & {[16] } \text { Oliveira PE. Asymptotics for Associated Random Variables. Springer;2012. } \\ & {[17] } \text { Birkel, T. A note on the strong law of large numbers for positively dependent random } \\ & \text { variables. Statist. Probab. Lett. 1989;7:17-20. } \\ & {[18] } \text { Shen Y, Wang X, Yang W, Hu S. Almost sure convergence theorem and strong stabi- } \\ & \text { lity for weighted sums of NSD random variables. Acta Mathematica Sinica, English Series } \\ & 2013 ; 29: 743-756 . \\ & {[19] } \text { Prakasa Rao, BLS. Hajek-Renyi-type inequality for associated sequences. Statist. Probab. } \\ & \text { Lett. 2002;57:139-143 }\end{aligned}$ $\begin{aligned} & \\ & {[15] } \text { He D, Xu K, Xu X. Random Weighting Empirical Distribution Function and its Applications } \\ & \text { to Goodness-of-Fit Testing. Comm. Statist. Simulation Comput. 2015;44:1441-1452. } \\ & {[16] } \text { Oliveira PE. Asymptotics for Associated Random Variables. Springer;2012. } \\ & {[17] } \text { Birkel, T. A note on the strong law of large numbers for positively dependent random } \\ & \text { variables. Statist. Probab. Lett. 1989;7:17-20. } \\ & {[18] } \text { Shen Y, Wang X, Yang W, Hu S. Almost sure convergence theorem and strong stabi- } \\ & \text { lity for weighted sums of NSD random variables. Acta Mathematica Sinica, English Series } \\ & 2013 ; 29: 743-756 . \\ & {[19] } \text { Prakasa Rao, BLS. Hajek-Renyi-type inequality for associated sequences. Statist. Probab. } \\ & \text { Lett. 2002;57:139-143 }\end{aligned}$

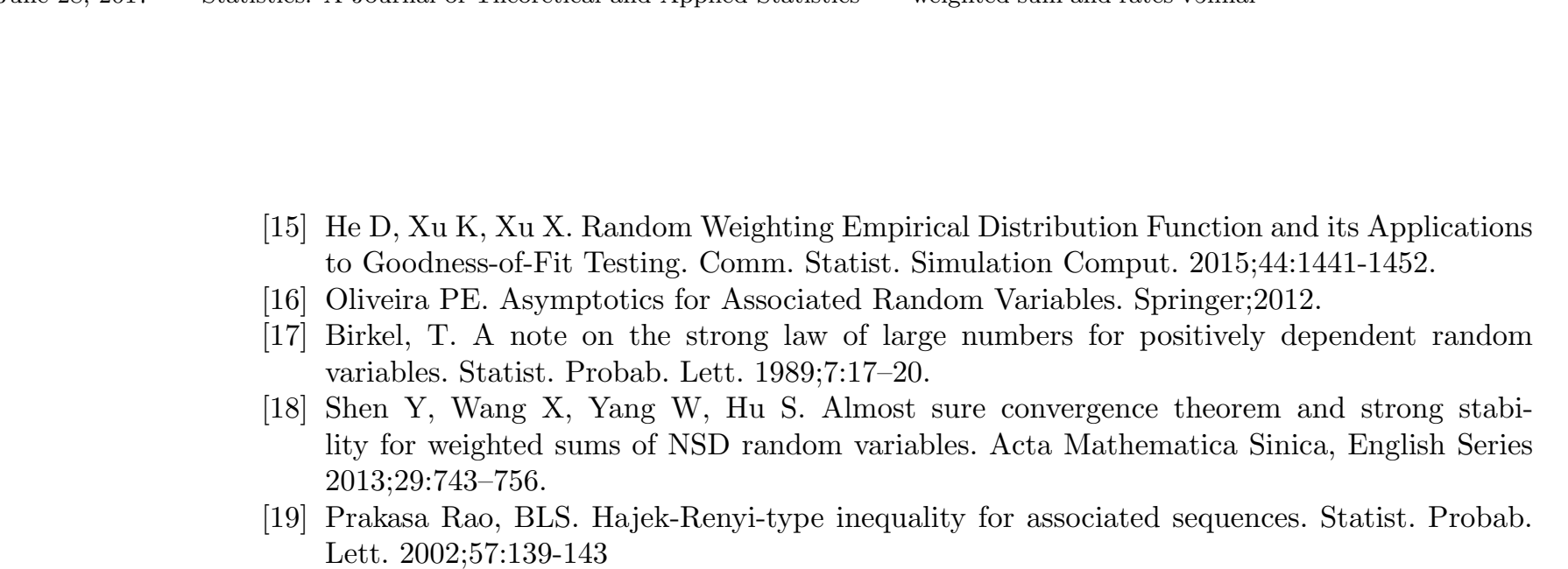
$\begin{aligned} & \\ & {[15] } \text { He D, Xu K, Xu X. Random Weighting Empirical Distribution Function and its Applications } \\ & \text { to Goodness-of-Fit Testing. Comm. Statist. Simulation Comput. 2015;44:1441-1452. } \\ & {[16] } \text { Oliveira PE. Asymptotics for Associated Random Variables. Springer;2012. } \\ & {[17] } \text { Birkel, T. A note on the strong law of large numbers for positively dependent random } \\ & \text { variables. Statist. Probab. Lett. 1989;7:17-20. } \\ & {[18] } \text { Shen Y, Wang X, Yang W, Hu S. Almost sure convergence theorem and strong stabi- } \\ & \text { lity for weighted sums of NSD random variables. Acta Mathematica Sinica, English Series } \\ & 2013 ; 29: 743-756 . \\ & {[19] } \text { Prakasa Rao, BLS. Hajek-Renyi-type inequality for associated sequences. Statist. Probab. } \\ & \text { Lett. 2002;57:139-143 }\end{aligned}$

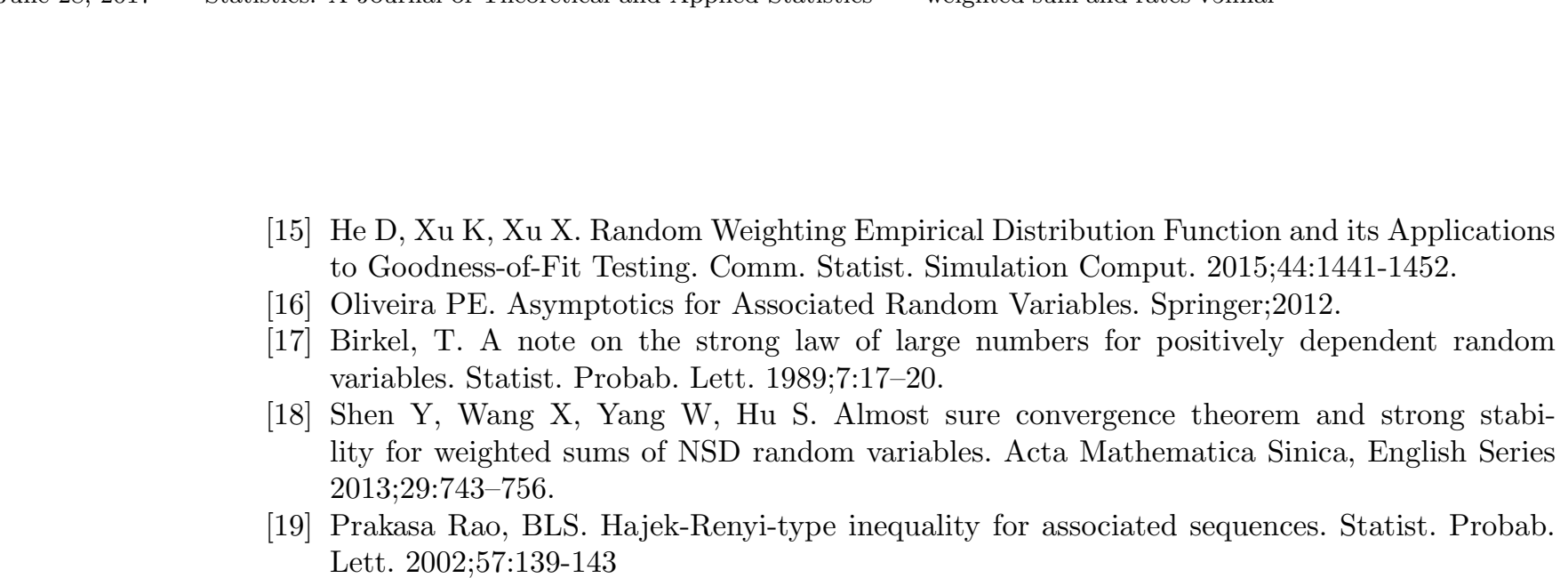
$\begin{aligned} & \\ {[15] } & \text { He D, Xu K, Xu X. Random Weighting Empirical Distribution Function and its Applications } \\ & \text { to Goodness-of-Fit Testing. Comm. Statist. Simulation Comput. 2015;44:1441-1452. } \\ {[16] } & \text { Oliveira PE. Asymptotics for Associated Random Variables. Springer;2012. } \\ {[17] } & \text { Birkel, T. A note on the strong law of large numbers for positively dependent random } \\ & \text { variables. Statist. Probab. Lett. 1989;7:17-20. } \\ {[18] } & \begin{array}{l}\text { Shen Y, Wang X, Yang W, Hu S. Almost sure convergence theorem and strong stabi- } \\ \text { lity for weighted sums of NSD random variables. Acta Mathematica Sinica, English Series } \\ 2013 ; 29: 743-756 .\end{array} \\ {[19] } & \begin{array}{l}\text { Prakasa Rao, BLS. Hajek-Renyi-type inequality for associated sequences. Statist. Probab. } \\ \text { Lett. 2002;57:139-143 }\end{array}\end{aligned}$

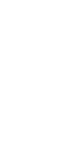

(n)

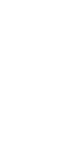
(1) 